\title{
Object Allocation via Deferred-Acceptance: Strategy-Proofness and Comparative Statics*
}

\author{
Lars Ehlers ${ }^{\dagger} \quad$ Bettina Klaus ${ }^{\ddagger}$
}

March 2016

\begin{abstract}
We study the problem of assigning indivisible and heterogenous objects to agents. Each agent receives at most one object and monetary compensations are not possible. We consider mechanisms satisfying a set of basic properties (unavailable-type-invariance, individual-rationality, weak non-wastefulness, or truncation-invariance).

In the house allocation problem, deferred- acceptance (DA)-mechanisms allocate objects based on exogenously fixed priorities over agents. We show that DA-mechanisms are characterized by our basic properties and (i) strategy-proofness and population-monotonicity or (ii) strategy-proofness and resource-monotonicity.

Once we allow for multiple identical copies of objects, on the one hand the first characterization breaks down and there are unstable mechanisms satisfying our basic properties and (i) strategy-proofness and population-monotonicity. On the other hand, our basic properties and (ii) strategy-proofness and resource-monotonicity characterize (the most general) class of DA-mechanisms based on objects' fixed choice functions that are acceptant, monotonic, substitutable, and consistent.
\end{abstract}

JEL Classification: D63, D70.

Keywords: indivisible objects allocation, deferred-acceptance-algorithm, strategy-proofness, resource-monotonicity, population-monotonicity.

\footnotetext{
${ }^{*}$ We are grateful to two anonymous referees, Battal Doğan, and Jan Christoph Schlegel for their helpful comments and suggestions. Lars Ehlers acknowledges financial support from the SSHRC (Canada) and the FRQSC (Québec) and Bettina Klaus acknowledges financial support from the Swiss National Science Foundation (SNSF).

${ }^{\dagger}$ Département de Sciences Économiques and CIREQ, Université de Montréal, Montréal, Québec H3C 3J7, Canada; e-mail: lars.ehlers@umontreal.ca.

${ }^{\ddagger}$ Corresponding author: Faculty of Business and Economics, University of Lausanne, Internef 538, CH-1015, Lausanne, Switzerland.; e-mail: bettina.klaus@unil.ch.
} 


\section{Introduction}

We study the simple model of assigning indivisible and heterogenous objects (e.g., houses, jobs, offices, school or university admissions etc.) to agents. In many real-life applications economists have recommended practitioners to use (variants of) the deferred-acceptance (DA)-algorithm to assign the objects to agents. Since in our model only one side of the market consists of agents and the other of objects, by DA-algorithm we refer to the agent-proposing DA-algorithm. For the DA-algorithm to be well-defined, objects need to be endowed with priorities over agents in order to determine which agents to reject in case of too many applications. When at most one copy of each object is available, priorities are given by strict orders of agents, and a priority structure is a collection of strict orders, one for each object. Once multiple identical copies of an object are available, priorities are given by choice functions, which choose for any set of agents wanting an object some agents to receive one each (no more than the number of available copies). Choice structures are a collection of choice functions, one for each object. Recent papers (Ehlers et al., 2014; Kamada and Kojima, 2015) constructed choice structures taking several important features from applications into account (like controlled school choice constraints or regional caps and target capacities) and then applied the DA-algorithm based on those constructed choice structures.

One key reason for applying the DA-algorithm in real life is that it is strategy-proof if the choice structure satisfies certain conditions. In addition, economists have advocated the attractive comparative statics properties of the DA-algorithm (e.g., Crawford, 1991, Hatfield and Milgrom, 2005, and Chambers and Yenmez, 2014): (i) population-monotonicity: once some agents leave, all remaining agents weakly benefit from this decrease in competition and (ii) resource-monotonicity: once more objects become available, all agents weakly benefit from this increase in resources. Our main results characterize responsive DA-algorithms and choice-based DA-algorithms via a set of basic properties in conjunction with strategy-proofness and one of the two comparative statics properties.

We first go back one step and consider the house allocation model $^{1}$ where at most one copy of each object is available. We show that in the house allocation model DA-mechanisms with strict priority structures are characterized (in the fixed resources model) by our basic properties, population-monotonicity, and strategy-proofness. However, this result does not hold in the general model where multiple identical copies of an object may be available and even if we add truncationinvariance (the chosen allocation remains unchanged if an agent truncates his preference below his assigned object): there are unstable mechanisms satisfying all our basic properties, populationmonotonicity, strategy-proofness, and truncation-invariance. In addition, all these properties minus strategy-proofness do not imply stability of the mechanism: the Boston mechanism satisfies all these properties except strategy-proofness (but the Boston mechanism is not stable).

\footnotetext{
${ }^{1}$ The search for "good" mechanisms to solve house allocation problems has been the subject of various contributions (among others): Ehlers (2002), Ehlers and Klaus (2004, 2007, 2011), Kesten (2009), and Pápai (2000).
} 
Considering the house allocation model with a fixed population and variable resources, our basic properties, resource-monotonicity, and strategy-proofness do not characterize responsive DAmechanisms. However, adding truncation-invariance to these properties yields another characterization of responsive DA-mechanism via our basic properties, resource-monotonicity, strategyproofness, and truncation-invariance. In the general model our main result characterizes choicebased DA-algorithms via a set of basic properties in conjunction with resource-monotonicity, strategy-proofness, and truncation-invariance. In addition, we show that any mechanism satisfying all these properties except strategy-proofness must be stable, i.e., we must be able to construct a choice structure such that for any problem the mechanism chooses an allocation that is stable with respect to this choice structure.

On the one hand, in the house allocation model population-monotonicity is the "stronger" comparative statics requirement because it characterizes responsive DA-mechanisms with our basic properties and strategy-proofness whereas resource-monotonicity does not (without adding truncation-invariance). On the other hand, in the multi-unit allocation model resourcemonotonicity is the "stronger" comparative statics requirement because it characterizes choicebased DA-mechanisms with our basic properties, strategy-proofness and truncation-invariance whereas population-monotonicity does not.

Therefore, in the general model, choice-based DA-mechanisms are the unique recommendation once we insist in addition to our basic properties on resource-monotonicity, populationmonotonicity, and strategy-proofness. Once we drop one of the key properties, choice-based DAmechanisms remain no longer the unique recommendation. This result advocates the DA-algorithm in applications in a stronger fashion than the usual ones whereby a mechanism is advocated because of its properties. The basic properties we use in our characterizations are satisfied by any real-life mechanism we are aware of, e.g., priority mechanisms, the Boston mechanism, linear programming mechanisms, the top trading cycles mechanism, the objects-proposing DA-algorithm etc.

Kojima and Manea (2010) were the first to obtain a characterization of choice-based DAmechanisms in the general model. They provided two characterizations, (a) one using the two properties of non-wastefulness and "individually rational monotonicity" and (b) one using the three properties of non-wastefulness, population-monotonicity, and "weak Maskin monotonicity". Whereas non-wastefulness is a basic requirement, "individual rational monotonicity" and "weak Maskin monotonicity" are new axioms that are arguably more difficult to explain to school boards in policy debates. Ehlers and Klaus (2014) characterize the smaller class of responsive DA-mechanisms whereby each choice function is based on a strict order and chooses from any set of agents the $k$ most preferred elements. In their main result, "two-agent consistent conflict resolution" plays a key role which says that at maximal conflict situations always the same agent should win the conflict (or receive the object). This property is violated by some choice-based DA-mechanisms. Furthermore, in contrast to these characterizations, we show that our main result minus strategy-proofness gives 
us stability, i.e., any such mechanism must be stable with respect to a given choice structure. Note that in all these contributions priorities are derived from a mechanism via a set of properties. Other papers take exogenous priorities as given and impose properties on the mechanism using these exogenous priorities. Balinski and Sönmez (1999) and Morrill (2013) are example of this approach and characterize the deferred-acceptance mechanisms based on "responsive" priorities and "substitutable" priorities.

The paper is organized as follows. In Section 2 we introduce our general object allocation model, properties of mechanisms, and the classes of deferred-acceptance-mechanisms that are either based on acceptant and responsive priority structures or on acceptant, monotonic, substitutable, and consistent choice structures over sets of agents. In Section 3 we state our characterization of responsivedeferred-acceptance mechanisms in the house allocation model using population-monotonicity and show how some previous and some new results are implied. Section 4 contains our main result (Theorem 3) for the general object allocation model using resource-monotonicity, a characterization of the class of choice-deferred-acceptance-mechanisms (where the choice structure satisfies acceptance, monotonicity, substitutability, and consistency). Section 5 concludes. All proofs and the independences of properties in our characterizations can be found in the Appendix.

\section{Object Allocation}

\subsection{The Model and Notation}

Let $^{2} N$ denote a finite set of agents, $|N| \geq 2$. We define the set of all nonempty subsets of $N$ by $\mathcal{N} \equiv\{S: S \subseteq N$ and $S \neq \emptyset\}$. Let $O$ denote a set of potential (real) object types or types for short. We assume that $O$ contains at least two elements and that $O$ is finite. ${ }^{3}$ Not receiving any real object is called "receiving the null object." Let $\emptyset$ represent the null object.

For a given set of agents $S \in \mathcal{N}$, each agent $i \in S$ is equipped with a preference relation $R_{i}$ over all types $O \cup\{\emptyset\}$. The preference relation $R_{i}$ is strict, i.e., $R_{i}$ is a linear order over $O \cup\{\emptyset\}$. Given $x, y \in O \cup\{\emptyset\}, x P_{i} y$ means that agent $i$ strictly prefers $x$ to $y$ (and $x \neq y$ ) and $x R_{i} y$ means that agent $i$ weakly prefers $x$ to $y$ (i.e., $x P_{i} y$ or $x=y$ ). Let $\mathcal{R}$ denote the set of all preferences over $O \cup\{\emptyset\}$, and $\mathcal{R}^{S}$ the set of all (preference) profiles $R=\left(R_{i}\right)_{i \in S}$ such that for all $i \in S, R_{i} \in \mathcal{R}$.

Given $R \in \mathcal{R}^{S}$ and $M \subseteq S$, let $R_{M}$ denote the profile $\left(R_{i}\right)_{i \in M}$. It is the restriction of $R$ to the set of agents $M$. We also use the notation $R_{-M}=R_{S \backslash M}$ and $R_{-i}=R_{S \backslash\{i\}}$ (whenever the set of agents $S$ has been fixed and no confusion can arise). Given $O^{\prime} \subseteq O \cup\{\emptyset\}$, let $\left.R_{i}\right|_{O^{\prime}}$ denote the restriction of $R_{i}$ to $O^{\prime}$ and $\left.R\right|_{O^{\prime}}=\left(\left.R_{i}\right|_{O^{\prime}}\right)_{i \in S}$. Given $i \in S$ and $R_{i} \in \mathcal{R}$, type $x \in O$ is acceptable under $R_{i}$ if $x P_{i} \emptyset$. Let $A\left(R_{i}\right)=\left\{x \in O: x P_{i} \emptyset\right\}$ denote the set of acceptable types under $R_{i}$.

\footnotetext{
${ }^{2}$ Our allocation model is identical to that described in Ehlers and Klaus (2014).

${ }^{3}$ Our results remain unchanged when $O$ is infinite. For expositional convenience, finiteness of $O$ is assumed.
} 
For each type $x \in O$, at most $\bar{q}_{x} \in \mathbb{N}$ copies are available in any economy with $1 \leq \bar{q}_{x} \leq|N|{ }^{4}$ Let $q_{x} \in\left\{0,1, \ldots, \bar{q}_{x}\right\}$ denote the number of available objects or the capacity of type $x$. Let $q=\left(q_{x}\right)_{x \in O}$ denote a capacity vector and $\mathcal{Q}=\times_{x \in O}\left\{0,1, \ldots, \bar{q}_{x}\right\}$ denote the set of all capacity vectors. The null object is always available without scarcity and therefore we set $q_{\emptyset}=\infty$. Given a capacity vector $q$, let $O_{+}(q)=\left\{x \in O: q_{x}>0\right\}$ denote the set of available real types under $q$. The set of available types is $O_{+}(q) \cup\{\emptyset\}$ and includes the null object

An (allocation) problem (with capacity constraints) consists of a set of agents $S \in \mathcal{N}$, a preference profile $R \in \mathcal{R}^{S}$, and a capacity vector $q$. We denote a problem by $(R, q)$ and the set of all problems by $\left(\cup_{S \in \mathcal{N}} \mathcal{R}^{S}\right) \times \mathcal{Q}$.

Given $S \in \mathcal{N}$ and $q \in \mathcal{Q}$, each agent $i$ is allocated exactly one object of a type in $O \cup\{\emptyset\}$ taking capacity constraints into account. An allocation ( for $S$ and $q$ ) is a list $a=\left(a_{i}\right)_{i \in S}$ such that for all $i \in S, a_{i} \in O \cup\{\emptyset\}$, and any real type $x \in O$ is not assigned more than $q_{x}$ times, i.e., for all $x \in O$, $\left|\left\{i \in S: a_{i}=x\right\}\right| \leq q_{x}$. Note that $\emptyset$, the null object, can be assigned to any number of agents and that not all real objects have to be assigned. Let $a(x)=\left\{i \in S: a_{i}=x\right\}$ denote the set of agents who are assigned type $x$ under $a$. Let $\mathcal{A}(S, q)$ denote the set of all allocations for $S$ and $q$, and $\mathcal{A}=\cup_{S \in \mathcal{N}} \cup_{q \in \mathcal{Q}} \mathcal{A}(S, q)$ the set of all allocations.

A mechanism is a function $\varphi:\left(\cup_{S \in \mathcal{N}} \mathcal{R}^{S}\right) \times \mathcal{Q} \rightarrow \mathcal{A}$ such that for all $S \in \mathcal{N}$, all $R \in \mathcal{R}^{S}$ and all $q \in \mathcal{Q}, \varphi(R, q) \in \mathcal{A}(S, q)$. Given $i \in S$, we call $\varphi_{i}(R, q)$ the allotment of agent $i$ at $\varphi(R, q)$.

\subsection{Properties of Mechanisms}

A natural requirement for a mechanism is that the chosen allocation depends only on preferences over the set of available types. Given a capacity vector $q$, a type $x$ is unavailable if $q_{x}=0$.

Unavailable-Type-Invariance: For all $S \in \mathcal{N}$, all $(R, q) \in \mathcal{R}^{S} \times \mathcal{Q}$, and all $R^{\prime} \in \mathcal{R}^{S}$ such that $\left.R\right|_{O_{+}(q) \cup\{\emptyset\}}=\left.R^{\prime}\right|_{O_{+}(q) \cup\{\emptyset\}}, \varphi(R, q)=\varphi\left(R^{\prime}, q\right) .^{5}$

By individual-rationality each agent should weakly prefer his allotment to the null object (which may represent an outside option such as off-campus housing in the context of university housing allocation, or private schools or home schooling in the context of student placement in public schools).

Individual-Rationality: For all $S \in \mathcal{N}$, all $(R, q) \in \mathcal{R}^{S} \times \mathcal{Q}$, and all $i \in S, \varphi_{i}(R, q) R_{i} \emptyset$.

\footnotetext{
${ }^{4}$ By introducing "global upper bounds" via $\bar{q}_{x}(x \in O)$ we can, for instance, specify the so-called house allocation model where at most one object of each type is available, i.e., for all $x \in O, \bar{q}_{x}=1$.

${ }^{5}$ Unavailable-type-invariance is similar to the independence if irrelevant commodities (IIC) property for social ordering functions introduced by Fleurbaey and Tadenuma (2007). It is important to note that "IIC is similar to the well known condition proposed by Arrow (1951), independence of irrelevant alternatives (IIA), but it turns out to be much weaker and, we believe, much less controversial." In Ehlers and Klaus (2004, 2011) we refer to unavailable-type-invariance as independence of irrelevant objects.
} 
Next, we introduce two properties that require a mechanism to not waste any resources. First, non-wastefulness (Balinski and Sönmez, 1999) requires that no agent prefers an available object that is not assigned to his allotment.

Non-Wastefulness: For all $S \in \mathcal{N}$, all $(R, q) \in \mathcal{R}^{S} \times \mathcal{Q}$, all $x \in O_{+}(q)$, and all $i \in N$, if $x P_{i} \varphi_{i}(R, q)$, then $\left|\left\{j \in S: \varphi_{j}(R, q)=x\right\}\right|=q_{x}$.

Next, we weaken non-wastefulness by requiring that no agent receives the null object while he prefers an available object that is not assigned.

Weak Non-Wastefulness: For all $S \in \mathcal{N}$, all $(R, q) \in \mathcal{R}^{S} \times \mathcal{Q}$, all $x \in O_{+}(q)$, and all $i \in S$, if $x P_{i} \varphi_{i}(R, q)$ and $\varphi_{i}(R, q)=\emptyset$, then $\left|\left\{j \in S: \varphi_{j}(R, q)=x\right\}\right|=q_{x}$.

Weak non-wastefulness is a limited efficiency requirement. For example, suppose that a central agency registers all agents who did not receive anything (or are unemployed) and all those agents report all real types (or jobs) which are acceptable to them. Then it should not be the case that some agent who receives nothing prefers one of the available real objects (or available jobs) to the null object.

Of course, no resources are wasted if a mechanism is (Pareto) efficient.

Efficiency: For all $S \in \mathcal{N}$ and all $(R, q) \in \mathcal{R}^{S} \times \mathcal{Q}$, there exists no feasible allocation $a \in \mathcal{A}(S, q)$ such that for all $i \in S, a_{i} R_{i} \varphi_{i}(R, q)$, and for some $j \in S, a_{j} P_{j} \varphi_{j}(R, q)$.

Note that efficiency implies individual-rationality and (weak) non-wastefulness.

When the set of objects varies, another natural requirement is resource-monotonicity. As already explained in the Introduction, this is a widely used solidarity property introduced by Chun and Thomson (1988) and it describes the effect of a change in the available resources on the welfare of the agents. A mechanism is resource-monotonic if the availability of more real objects has a (weakly) positive effect on all agents.

Resource-Monotonicity: For all $S \in \mathcal{N}$, all $R \in \mathcal{R}^{S}$, and all $q, q^{\prime} \in \mathcal{Q}$, if $q_{x} \leq q_{x}^{\prime}$ for all $x \in O$, then for all $i \in S, \varphi_{i}\left(R, q^{\prime}\right) R_{i} \varphi_{i}(R, q)$.

Remark 1 (Resource-Monotonicity implies Individual-Rationality).

Let $q^{0} \in \mathcal{Q}$ be such that for all $x \in O, q_{x}^{0}=0$. Note that for all $S \in \mathcal{N}$ and all $R \in \mathcal{R}^{S}$, $\varphi_{i}\left(R, q^{0}\right)=\emptyset$. Therefore, resource-monotonicity implies individual-rationality (because then for all $q \in \mathcal{Q}, q_{x}^{0} \leq q_{x}$, and $\varphi_{i}(R, q) R_{i} \emptyset$ for all $\left.i \in S\right){ }^{6}$

Another solidarity property concerning variations of the population of agents is populationmonotonicity; it requires that as the set of agents becomes larger, the initially present agents all get (weakly) worse off. This property goes back to Thomson (1983), who also presents a survey of population monotonicity in various economic models (Thomson, 1995).

\footnotetext{
${ }^{6}$ We thank Battal Doğan for pointing out that in our model resource-monotonicity implies individual-rationality.
} 
Population-Monotonicity: For all $T \subseteq S \in \mathcal{N}$ and all $(R, q) \in \mathcal{R}^{S} \times \mathcal{Q}$, we have for all $i \in T$, $\varphi_{i}\left(R_{T}, q\right) R_{i} \varphi_{i}(R, q)$.

Many mechanisms that are used in real life ignore agents' preferences below their allotments (e.g., any mechanism based on or equivalent to the famous deferred-acceptance-algorithm or the so-called priority mechanisms, Roth and Sotomayor, 1990, Sections 5.4.1 and 5.5.1). That is, an allocation does not change if an agent changes his reported preferences below his allotment. We formulate a weaker version of this invariance property by restricting agents' changes below their allotments to truncations.

A truncation strategy is a preference relation that ranks the real types in the same way as the corresponding original preference relation and each real type which is acceptable under the truncation strategy is also acceptable under the original preference relation. Formally, given $S \in \mathcal{N}$, $i \in S$ and $R_{i} \in \mathcal{R}$, a strategy $\bar{R}_{i} \in \mathcal{R}$ is a truncation (strategy) of $R_{i}$ if (t1) $\left.\bar{R}_{i}\right|_{O}=\left.R_{i}\right|_{O}$ and (t2) $A\left(\bar{R}_{i}\right) \subseteq A\left(R_{i}\right)$. Loosely speaking, a truncation strategy of $R_{i}$ is obtained by moving the null object "up."

If an agent truncates his preference relation in a way such that his allotment remains acceptable under the truncated preference relation, then truncation-invariance requires that the allocation is the same under both profiles. The property is quite natural on its own in the sense that the chosen allocations do not depend on where any agent, who receives a real object, ranks the null object below his allotment.

Truncation-Invariance: For all $S \in \mathcal{N}$, all $(R, q) \in \mathcal{R}^{S} \times \mathcal{Q}$, all $i \in S$, and all $\bar{R}_{i} \in \mathcal{R}$, if $\bar{R}_{i}$ is a truncation of $R_{i}$ and $\varphi_{i}(R, q)$ is acceptable under $\bar{R}_{i}\left(i . e ., \varphi_{i}(R, q) \in A\left(\bar{R}_{i}\right)\right)$, then $\varphi\left(\left(\bar{R}_{i}, R_{-i}\right), q\right)=\varphi(R, q)$.

The well-known non-manipulability property strategy-proofness requires that no agent can ever benefit from misrepresenting his preferences.

Strategy-Proofness: For all $S \in \mathcal{N}$, all $(R, q) \in \mathcal{R}^{S} \times \mathcal{Q}$, all $i \in S$, and all $\bar{R}_{i} \in \mathcal{R}, \varphi_{i}(R, q) R_{i}$ $\varphi_{i}\left(\left(\bar{R}_{i}, R_{-i}\right), q\right)$.

The following strengthening of strategy-proofness requires that no group of agents can ever benefit by misrepresenting their preferences.

Group-Strategy-Proofness: For all $S \in \mathcal{N},(R, q) \in \mathcal{R}^{S} \times \mathcal{Q}$, all $M \subseteq S(M \neq \emptyset)$, and all $\bar{R}_{M} \in \mathcal{R}^{M}$, if for all $i \in M, \varphi_{i}\left(\left(\bar{R}_{M}, R_{-M}\right), q\right) R_{i} \varphi_{i}(R, q)$, then for all $i \in M, \varphi_{i}\left(\left(\bar{R}_{M}, R_{-M}\right), q\right)=$ $\varphi_{i}(R, q)$.

\subsection{Stability and Deferred Acceptance}

\subsection{1 "Classic" Stability}

In this subsection, let $S \in \mathcal{N}$ be fixed and consider fixed-population mechanisms $\varphi: \mathcal{R}^{S} \times \mathcal{Q} \rightarrow \mathcal{A}$. 
Given type $x \in O$, let $\succ_{x}$ denote a priority ordering on $S$, e.g., $\succ_{x}: 12 \ldots|S|$ means that agent 1 has higher priority for type $x$ than agent 2, who has higher priority for type $x$ than agent 3 , etc. Let $\succ \equiv\left(\succ_{x}\right)_{x \in O}$ denote a priority structure for $S$. Then, given a priority structure $\succ$ for $S$, and a problem $(R, q) \in \mathcal{R}^{S} \times \mathcal{Q}$, we can interpret $(R, q, \succ)$ as a responsive many-to-one matching market (Gale and Shapley, 1962; Roth and Sotomayor, 1990) where the set of agents $S$ corresponds to the set of students, the set of available real types $O_{+}(q)$ corresponds to the set of colleges, preferences $\left.R\right|_{O_{+}(q) \cup\{\emptyset\}}$ correspond to students' preferences over colleges and being unmatched, and the priority structure $\left(\succ_{x}\right)_{x \in O}$ corresponds to colleges' responsive preferences over students in $S$.

Formally, a priority relation $P_{x}$ on $\left\{S^{\prime} \subseteq S: S^{\prime} \neq \emptyset \&\left|S^{\prime}\right| \leq q_{x}\right\}$ is responsive to $\succ_{x}$ if the following two conditions hold: (r1) for all $\emptyset \neq S^{\prime} \subseteq S$ such that $\left|S^{\prime}\right|<q_{x}$ and all $i \in S \backslash S^{\prime}$, $S^{\prime} \cup\{i\} P_{x} S^{\prime}$ and (r2) for all $\emptyset \neq S^{\prime} \subseteq S$ such that $\left|S^{\prime}\right|<q_{x}$ and all $i, j \in S \backslash S^{\prime},\left(S^{\prime} \cup\{i\}\right) P_{x}\left(S^{\prime} \cup\{j\}\right)$ if and only if $i \succ_{x} j$.

Stability is an important requirement for many real-life matching markets and it will turn out to be essential in our context of allocating indivisible objects to agents as well.

$\succ$-Stability: Given $(R, q) \in \mathcal{R}^{S} \times \mathcal{Q}$, an allocation $a \in \mathcal{A}(S, q)$ is $\succ$-stable if

(s1) it is individually-rational and non-wasteful, i.e., there exists no agent-type pair $(i, x) \in S \times$ $\left(O_{+}(q) \cup\{\emptyset\}\right)$ such that $x P_{i} a_{i}$ and $\left|\left\{j \in S: a_{j}=x\right\}\right|<q_{x}$ and

(s2) there are no blocking pairs, i.e., there exists no agent-type pair $(i, x) \in S \times O_{+}(q)$ such that $x P_{i} a_{i}$ and there exists $k \in N$ such that $a_{k}=x$ and $i \succ_{x} k$.

Note that $\succ$-stability implies individual-rationality and (weak) non wastefulness, but it does not imply efficiency. Fixed-population mechanism $\varphi$ is $\succ$-stable if for all $(R, q) \in \mathcal{R}^{S} \times \mathcal{Q}, \varphi(R, q)$ is $\succ$-stable.

$\succ$-Agent-Proposing Deferred-Acceptance-Algorithm: given priority structure $\succ$ and problem $(R, q) \in \mathcal{R}^{S} \times \mathcal{Q}$, the agent-proposing deferred-acceptance-algorithm (Gale and Shapley, 1962) is defined as follows:

- at the first step of the deferred-acceptance-algorithm, every agent applies to his favorite type in $O_{+}(q) \cup\{\emptyset\}$. For each available real type $x \in O_{+}(q)$, the $q_{x}$ applicants who have $\succ_{x}$-highest priority for $x$ are placed on the waiting list of $x$ (all if there are fewer than $q_{x}$ applicants), and all others are rejected. The null object $\emptyset$ accepts all agents.

- At the $r$-th step of the deferred-acceptance-algorithm, those applicants who were rejected at step $r-1$ apply to their next favorite type in $O_{+}(q) \cup\{\emptyset\}$. For each available real type $x \in O_{+}(q)$, the $q_{x}$ applicants among the new applicants and those on the waiting list at the end of Step $r-1$ who have the $\succ_{x}$-highest priority for $x$ are placed on the (updated) waiting list of $x$ (all if there are fewer than $q_{x}$ applicants), and all others are rejected. The null object $\emptyset$ accepts all agents. 
The deferred-acceptance-algorithm terminates when every agent is on a waiting list. Once the algorithm ends, available real objects are assigned to the agents on the available real type waiting lists (all other agents were accepted by and receive the null object) and the resulting allocation is the agent-optimal stable allocation for the responsive many-to-one matching market $(R, q, \succ)$, denoted by $D A^{\succ}(R, q)$.

Responsive-Deferred-Acceptance-Mechanisms: A fixed-population mechanism $\varphi: \mathcal{R}^{S} \times \mathcal{Q} \rightarrow$ $\mathcal{A}$ is a responsive-deferred-acceptance-mechanism if there exists a strict priority structure $\succ$ such that for all $(R, q) \in \mathcal{R}^{S} \times \mathcal{Q}, \varphi(R, q)=D A^{\succ}(R, q)$.

\subsubsection{Choice Function Stability}

In this subsection we return to our variable population setup and instead of using responsive priorities over sets of agents, we use a choice function approach. For any real type $x$, a choice function chooses for any set of agents and any number of available copies of $x$, a non-empty subset. The choice functions will be used by types to accept/reject agents in the agent-proposing deferredacceptance algorithm.

This approach has been proven to be useful to define priority relations on the power set of agents which are not necessarily responsive to a priority ordering but still guarantee the existence of stable allocations (see for instance, Ehlers et al., 2014, and Kamada and Kojima, 2015). An important role here plays substitutability defined below. Further conditions on the choice function are needed in order to guarantee strategy-proofness, resource-monotonicity, and/or population-monotonicity of the agent-proposing deferred-acceptance algorithm.

For any real type $x \in O$, let $\mathcal{C}_{x}: \mathcal{N} \times\left\{1, \ldots, \bar{q}_{x}\right\} \rightarrow \mathcal{N}$ denote a choice function such that for all $S \in \mathcal{N}$ and all $l \in\left\{1, \ldots, \bar{q}_{x}\right\}, \mathcal{C}_{x}(S, l) \subseteq S$ and $\mathcal{C}_{x}(S, l) \neq \emptyset$. We next list some properties of choice functions.

Acceptance: Choice function $\mathcal{C}_{x}$ is acceptant if for all $S \in \mathcal{N}$ and all $l \in\left\{1, \ldots, \bar{q}_{x}\right\},\left|\mathcal{C}_{x}(S, l)\right|=$ $\min \{|S|, l\}$.

Monotonicity: Choice function $\mathcal{C}_{x}$ is monotonic if for all $S \in \mathcal{N}$ and all $l \in\left\{2, \ldots, \bar{q}_{x}\right\}, \mathcal{C}_{x}(S, l-$ 1) $\subseteq \mathcal{C}_{x}(S, l)$.

Substitutability: Choice function $\mathcal{C}_{x}$ is substitutable if for all $S \in \mathcal{N}$, all $l \in\left\{1, \ldots, \bar{q}_{x}\right\}$, and all $i, j \in S$ with $i \neq j, i \in \mathcal{C}_{x}(S, l)$ implies $i \in \mathcal{C}_{x}(S \backslash\{j\}, l)$.

Consistency: Choice function $\mathcal{C}_{x}$ is consistent if for all $S, S^{\prime} \in \mathcal{N}$ and all $l \in\left\{1, \ldots, \bar{q}_{x}\right\}, \mathcal{C}_{x}(S, l) \subseteq$ $S^{\prime} \subseteq S$ implies $\mathcal{C}_{x}(S, l)=\mathcal{C}_{x}\left(S^{\prime}, l\right)$. 
Lemma 1. If choice function $\mathcal{C}_{x}$ satisfies acceptance and substitutability, then $\mathcal{C}_{x}$ satisfies consistency. ${ }^{7}$

Proof. Let $S, S^{\prime} \in \mathcal{N}$ and $l \in\left\{1, \ldots, \bar{q}_{x}\right\}$ be such that $\mathcal{C}_{x}(S, l) \subseteq S^{\prime} \subseteq S$. By substitutability, $\mathcal{C}_{x}(S, l) \subseteq \mathcal{C}_{x}\left(S^{\prime}, l\right)$. By acceptance, $\left|\mathcal{C}_{x}\left(S^{\prime}, l\right)\right| \leq\left|\mathcal{C}_{x}(S, l)\right|$ which implies $\mathcal{C}_{x}(S, l)=\mathcal{C}_{x}\left(S^{\prime}, l\right)$.

A choice structure is a profile of choice functions, i.e., $\mathcal{C}=\left(\mathcal{C}_{x}\right)_{x \in O}$. For all $S \in \mathcal{N}$ and all $l \in\{1, \ldots,|N|\}$, we define $\mathcal{C}_{\emptyset}(S, l)=S$ (the null object always chooses all agents). We say that choice structure $\mathcal{C}$ is acceptant / monotonic / substitutable / consistent if for all real types $x \in O$, $\mathcal{C}_{x}$ is acceptant / monotonic / substitutable / consistent.

$\mathcal{C}$-Stability: Given $S \in \mathcal{N}$ and $(R, q) \in \mathcal{R}^{S} \times \mathcal{Q}$, an allocation a $\in \mathcal{A}(S, q)$ is $\mathcal{C}$-stable if

(s1) it is individually-rational for both sides of the market, i.e., for all $i \in S, a_{i} R_{i} \emptyset$ and for all $x \in O_{+}(q) \cup\{\emptyset\}, \mathcal{C}_{x}\left(a(x), q_{x}\right)=a(x)$ and

(s2) there are no blocking pairs, i.e., there exists no agent-type pair $(i, x) \in S \times O_{+}(q)$ such that $x P_{i} a_{i}$ and $i \in \mathcal{C}_{x}\left(a(x) \cup\{i\}, q_{x}\right)$.

Note that $\mathcal{C}$-stability implies individual-rationality and (weak) non wastefulness, but it does not imply efficiency. Mechanism $\varphi$ is $\mathcal{C}$-stable if for all $S \in \mathcal{N}$ and $(R, q) \in \mathcal{R}^{S} \times \mathcal{Q}, \varphi(R, q)$ is $\mathcal{C}$-stable.

$\mathcal{C}$-Agent-Proposing Deferred-Acceptance-Algorithm: Given choice structure $\mathcal{C}$, a set of agents $S \in \mathcal{N}$, and a problem $(R, q) \in \mathcal{R}^{S} \times \mathcal{Q}$, the agent-proposing deferred-acceptance-algorithm is defined as follows:

- at the first step of the deferred-acceptance-algorithm, every agent applies to his favorite type in $O_{+}(q) \cup\{\emptyset\}$. For each available real type $x \in O_{+}(q)$, let $S_{x, 1}$ denote the set of agents who applied to real type $x$, applicants in $\mathcal{C}_{x}\left(S_{x, 1}, q_{x}\right)$ are placed on the waiting list of $x$, and all others are rejected. The null object $\emptyset$ accepts all agents.

- At the $r$-th step of the deferred-acceptance-algorithm, those applicants who were rejected at step $r-1$ apply to their next favorite type in $O_{+}(q) \cup\{\emptyset\}$. For each available real type $x \in O_{+}(q)$, let $S_{x, r}$ denote the set of new applicants and those on the waiting list at the end of Step $r-1$, applicants in $\mathcal{C}_{x}\left(S_{x, r}, q_{x}\right)$ are placed on the (updated) waiting list of $x$, and all others are rejected. The null object $\emptyset$ accepts all agents.

\footnotetext{
${ }^{7}$ Consistency is referred to as irrelevance of rejected contracts (IRC) by Aygün and Sönmez (2013). Note that acceptance implies the law of aggregate demand $(L A D): \mathcal{C}_{x}$ satisfies LAD if for all $S, S^{\prime} \in \mathcal{N}$ and all $l \in\left\{1, \ldots, \bar{q}_{x}\right\}$, $S^{\prime} \subseteq S$ implies $\left|\mathcal{C}_{x}\left(S^{\prime}, l\right)\right| \leq\left|\mathcal{C}_{x}(S, l)\right|$. Aygün and Sönmez (2013) state "Some of the analysis in Hatfield and Milgrom (2005) assumes the law of aggregate demand (LAD) condition in addition to the substitutes condition. These two conditions together imply the IRC condition, ...".
} 
The deferred-acceptance-algorithm terminates when every agent is on a waiting list. Once the algorithm ends, available real objects are assigned to the agents on the available real type waiting lists (all other agents were accepted by and receive the null object). Let $D A^{\mathcal{C}}(R, q)$ denote the allocation determined by the choice-based deferred-acceptance-algorithm when applied to $(R, q, \mathcal{C})$.

Hatfield and Milgrom (2005) and Aygün and Sönmez (2013) have showed that if $\mathcal{C}$ is acceptant, substitutable, and consistent, then $D A^{\mathcal{C}}(R, q)$ is $\mathcal{C}$-stable (and mechanism $D A^{\mathcal{C}}$ is strategy-proof). ${ }^{8}$

Choice-Deferred-Acceptance-Mechanisms: A mechanism $\varphi:\left(\cup_{S \in \mathcal{N}} \mathcal{R}^{S}\right) \times \mathcal{Q} \rightarrow \mathcal{A}$ is a choicedeferred-acceptance-mechanism if there exists a choice structure $\mathcal{C}$ such that for all $S \in \mathcal{N}$ and all $(R, q) \in \mathcal{R}^{S} \times \mathcal{Q}, \varphi(R, q)=D A^{\mathcal{C}}(R, q)$.

\section{Population-Monotonicity}

For the so-called house allocation model at most one copy of each real type is available. Hence, for all $x \in O, \bar{q}_{x}=1$. We denote the set of all house allocation capacity vectors by $\mathcal{Q}_{1}$.

We keep the set of types $O$ fixed and vary the set of agents to be $S \in \mathcal{N}$. Hence, a mechanism is a function $\varphi:\left(\cup_{S \in \mathcal{N}} \mathcal{R}^{S}\right) \rightarrow \mathcal{A}$ (with a fixed set of types $O$ ). The definitions of $\succ$-stability and the $\succ$-proposing deferred-acceptance-algorithm are as before with the understanding that $\succ$ is a priority structure for $N$ that induces corresponding priority structures for all $S \in \mathcal{N}$, i.e., if agent $i \in N$ has a higher priority for a type $x$ than agent $j \in N$, then for any set $S \in \mathcal{N}$ such that $i, j \in S$, agent $i$ again has a higher priority for type $x$ than agent $j$. The house allocation model (with fixed resources and a variable population) is identical to that described in Ehlers, Klaus, and Pápai (2002).

Theorem 1 (Responsive-DA-Mechanisms: Population-Monotonicity).

In the house allocation model (with fixed resources and a variable population), responsive-deferredacceptance-mechanisms are the only mechanisms satisfying individual-rationality, weak nonwastefulness, population-monotonicity, and strategy-proofness.

We prove Theorem 1 in Appendix A.1. We prove the independence of the properties in Theorem 1 in Appendix A.2.

Interestingly, in the house allocation model (with fixed resources and a variable population) all properties of Theorem 1 minus strategy-proofness do not imply $\succ$-stability (even if we added truncation-invariance): the so-called Boston (or immediate-acceptance) algorithm defines a mechanism that satisfies all properties of Theorem 1 (and truncation-invariance) but strategy-proofness and it is not $\succ$-stable for any priority structure $\succ$ (see also our later Remark 2).

\footnotetext{
${ }^{8}$ Earlier results for the existence of stable allocations under substitutability are Kelso and Crawford (1982) and Roth (1984).
} 
We introduce an acyclicity condition which equals Ergin's (2002) acyclicity condition for the general model when adapted to house allocation problems.

Cycles and Acyclicity: Given a priority structure $\succ$ a cycle consists of distinct $x, y \in O$ and $i, j, k \in S$ such that $i \succ_{x} j \succ_{x} k$ and $k \succ_{y} i$. A priority structure $\succ$ is acyclic if it has no cycles. A responsive-deferred-acceptance-mechanism is acyclic if the associated priority structure is acyclic.

Ergin (2002, Theorem 1) shows that the acyclicity of the priority structure $\succ$ is equivalent to efficiency or group-strategy-proofness of the induced responsive-deferred-acceptance-mechanism $D A^{\succ}$. Furthermore, with Kesten's (2009, Theorem 1) result it follows that for house allocation the class of efficient responsive-deferred-acceptance-mechanisms equals the class of restricted endowment inheritance rules in Ehlers, Klaus, and Pápai (2002). Then, Theorem 1 implies the following characterizations of the subclass of acyclic responsive-deferred-acceptance-mechanisms.

Corollary 1 (Acyclic Responsive-DA-Mechanisms: Population-Monotonicity).

(a) In the house allocation model (with fixed resources and a variable population), responsivedeferred-acceptance-mechanisms with acyclic priority structures are the only mechanisms satisfying efficiency, population-monotonicity, and strategy-proofness.

(b) In the house allocation model (with fixed resources and a variable population), responsivedeferred-acceptance-mechanisms with acyclic priority structures are the only mechanisms satisfying individual-rationality, weak non-wastefulness, population-monotonicity, and group-strategyproofness.

Corollary 1 (a) equals Ehlers, Klaus, and Pápai's (2002) Theorem 1. We prove Corollary 1 in Appendix A.3.

The following example demonstrates that Theorem 1 does not hold for our more general model where more than one copy of each object might be available. This is even true if we added truncation-invariance to the properties in Theorem 1 because the mechanism below satisfies truncation-invariance. In addition we show that the example below is not $\mathcal{C}$-stable with an acceptant choice structure $\mathcal{C}$, i.e., even the more general approach does not yield a positive result.

Example 1 (Theorem 1 does not generalize).

Let $N=\{1,2,3,4\}, O=\{x, y\}$, and there are two copies of $x$ and one copy of $y$ available, i.e., $q_{x}=2, q_{y}=1$ and $q=\left(q_{x}, q_{y}\right)$. We then denote a problem by $R \in \mathcal{R}^{S}(S \in \mathcal{N})$. The following mechanism $f$ satisfies individual-rationality, weak non-wastefulness, population-monotonicity, and strategy-proofness.

Let $\succ_{x}: 1234, \succ_{x}^{\prime}: 1243$, and $\succ_{y}: 1234$. Let $\succ=\left(\succ_{x}, \succ_{y}\right)$ and $\succ^{\prime}=\left(\succ_{x}^{\prime}, \succ_{y}\right)$. Given $R_{i}$, let top $\left(R_{i}\right)$ denote agent $i$ 's favorite object in $O \cup\{\emptyset\}$. Then, for each problem $R \in \mathcal{R}^{S}(S \in \mathcal{N})$,

$$
f(R)= \begin{cases}D A^{\succ^{\prime}}(R, q) & \text { if } \operatorname{top}\left(R_{1}\right)=\operatorname{top}\left(R_{2}\right)=y \text { and } \\ D A^{\succ}(R, q) & \text { otherwise. }\end{cases}
$$


Note that $f$ is not $\mathcal{C}$-stable with an acceptant choice structure $\mathcal{C}$ (and therefore, $f$ is not a responsivedeferred-acceptance-mechanism): suppose that $\mathcal{C}$ is an acceptant choice structure and $f$ is $\mathcal{C}$-stable; if $R \in \mathcal{R}^{N}$ is such that $R_{1}: y \emptyset x$ and $R_{2}=R_{3}=R_{4}: x \emptyset y$, then $f(R)=D A^{\succ}(R, q)$, i.e., agents 2 and 3 receive $x$ and agent 4 receives $\emptyset$ (not the desired $x$ ), implying $4 \notin \mathcal{C}_{x}(\{2,3,4\}, 2)$; if $R_{2}^{\prime}: y x \emptyset$ and $R^{\prime}=\left(R_{2}^{\prime}, R_{-2}\right)$, then $f\left(R^{\prime}\right)=D A^{\succ^{\prime}}\left(R^{\prime}, q\right)$, i.e., agents 2 and 4 receive $x$ and agent 3 receives $\emptyset$ (not the desired $x$ ), implying $3 \notin \mathcal{C}_{x}(\{2,3,4\}, 2)$; but now $3,4 \notin \mathcal{C}_{x}(\{2,3,4\}, 2)$ and $\mathcal{C}_{x}$ is not acceptant, a contradiction. Note that this argument also establishes that there does not exist a collection of acceptant choice structures $\left(\hat{\mathcal{C}}^{S}\right)_{S \in \mathcal{N}}$ (one for each $S \in \mathcal{N}$ ) such that for any $R \in \mathcal{R}^{S}$ $(S \in \mathcal{N}), f(R)$ is stable with respect to $\hat{\mathcal{C}}^{S}$ : we would have to have both $3,4 \notin \hat{\mathcal{C}}_{x}^{N}(\{2,3,4\}, 2)$ and $\hat{\mathcal{C}}_{x}^{N}$ is not acceptant, a contradiction.

It is easy to see that $f$ satisfies individual-rationality, weak non-wastefulness, and strategyproofness (and truncation-invariance).

For population-monotonicity, let $R \in \mathcal{R}^{N}$ and $T, S \in \mathcal{N}$ such that $T \varsubsetneqq S$. It suffices to check for possible violations of population-monotonicity when $f$ uses a different priority structure for $R_{S}$ and $R_{T}$. Hence, $1,2 \in S$, $\operatorname{top}\left(R_{1}\right)=\operatorname{top}\left(R_{2}\right)=y$, and $1 \notin T$ or $2 \notin T$. Thus, $f_{1}\left(R_{S}\right)=y$.

If $R_{2}: y \emptyset x$, then $f_{2}\left(R_{S}\right)=\emptyset$ and population-monotonicity is satisfied if 1 or 2 leaves problem $R_{S}$ (because both copies of $x$ are available for the remaining agent(s)). Let $R_{2}: y x \emptyset$. Then $f_{2}\left(R_{S}\right)=x$. If 1 leaves problem $R_{S}$ and $2 \in T$, then $f_{2}\left(R_{T}\right)=y$ and again population-monotonicity is satisfied (because both copies of $x$ are available for the remaining agent(s)). The case where 1 leaves problem $R_{S}$ and $2 \notin T$ is trivial. If 2 leaves problem $R_{S}$ and $1 \in T$, then $f_{1}\left(R_{T}\right)=y$ and again population-monotonicity is satisfied (because both copies of $x$ are available for the remaining agent(s)). The case where 2 leaves problem $R_{S}$ and $1 \notin T$ is trivial.

Note that Example 1 is with fixed resources and there are two copies of $x$ and one copy of $y$. Agents 1 and 2 have higher priority than agents 3 and 4 at any type, and the priority between 3 and 4 at $x$ is different when agents 1 and 2 rank $y$ first and when not. As we will see below, such an example is not possible for unavailable-type-invariance and resource-monotonicity. This is due to the fact that with variable resources, when agent 1 does not rank $x$ acceptable and all other agents rank $x$ acceptable, with two available copies of $x$ agents 2 and 3 must receive $x$ or agents 2 and 4 must receive $x$. But now when one copy of $y$ is added, then the priority between 3 and 4 for $x$ may change depending whether agents 1 and 2 rank $y$ first or not (here unavailable-type-invariance is used).

\section{Resource-Monotonicity}

Instead of population-monotonicity and unit capacities, we now consider resource-monotonicity and non-unit capacities with a fixed population and variable resources. Let $N$ be fixed and consider fixed-population mechanisms $\varphi: \mathcal{R}^{N} \times \mathcal{Q} \rightarrow \mathcal{A}$. 
We first show that unavailable-type-invariance, weak non-wastefulness, resource-monotonicity, and truncation-invariance imply stability with respect to some acceptant, monotonic, substitutable, and consistent choice structure.

Theorem 2 (Existence of a Choice Structure and Stability: Resource-Monotonicity). If a mechanism $\varphi$ satisfies unavailable-type-invariance, weak non-wastefulness, resourcemonotonicity, and truncation-invariance, then there exists a choice structure $\mathcal{C}$ satisfying acceptance, monotonicity, substitutability, and consistency such that $\varphi$ is $\mathcal{C}$-stable.

The main characterization for our general allocation model is as follows.

Theorem 3 (Choice-DA-Mechanisms: Resource-Monotonicity).

Choice-deferred-acceptance-mechanisms (where the choice structure satisfies acceptance, monotonicity, substitutability, and consistency) are the only mechanisms satisfying unavailable-typeinvariance, weak non-wastefulness, resource-monotonicity, truncation-invariance, and strategyproofness.

We prove Theorems 2 and 3 in Appendix A.4. We prove the independence of properties in Theorem 3 in Appendix A.5.

Note that the type-proposing choice-deferred-acceptance-mechanisms (where the choice structure satisfies acceptance, monotonicity, substitutability, and consistency) satisfy all properties of Theorem 2 and are $\mathcal{C}$-stable. However, they are not strategy-proof.

In the general setup Kumano (2009) introduced an acyclicity condition on choice structures which is necessary and sufficient for the induced choice-deferred-acceptance mechanisms to satisfy efficiency or group-strategy-proofness.

$*_{\text {-Cycles and }}^{*}$-Acyclicity: Given a choice structure $\mathcal{C}$, a ${ }^{*}$-cycle consists of distinct $x, y \in O$, $l \in\left\{1, \ldots, \bar{q}_{x}\right\}, m \in\left\{1, \ldots, \bar{q}_{y}\right\}$, distinct $i, j, k \in N$ and disjoint $S_{x}, S_{y} \subseteq N \backslash\{i, j, k\}$ such that (i) $j \notin \mathcal{C}_{x}\left(S_{x} \cup\{i, j\}, l\right), k \notin \mathcal{C}_{x}\left(S_{x} \cup\{j, k\}, l\right)$, and $i \notin \mathcal{C}_{y}\left(S_{y} \cup\{k, i\}, m\right)$ and (ii) both $\left|S_{x}\right|=l-1$ and

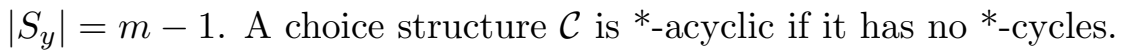

A choice-deferred-acceptance mechanism is *-acyclic if the associated choice structure is *acyclic.

The result below follows from Kumano (2009, Theorem 1) and Kojima and Manea (2010, Proposition 1).

Corollary 2 (Acyclic Choice-DA-Mechanisms Characterized: Resource-Monotonicity).

(a) *-Acyclic choice-deferred-acceptance-mechanisms are the only mechanisms satisfying unavailable-type-invariance, efficiency, resource-monotonicity, and truncation-invariance.

(b) *-Acyclic choice-deferred-acceptance-mechanisms are the only mechanisms satisfying weak nonwastefulness, resource-monotonicity, and group-strategy-proofness.

The proof of Corollary 2 is in Appendix A.6. 


\section{Conclusion}

\subsection{Unit Capacities}

First, note that for the house allocation model with fixed population and variable resources the following are immediate corollaries from Theorem 2 and Theorem 3.

Corollary 3 (Theorem 2 for House Allocation).

In the house allocation model (with a fixed population and variable resources), if a mechanism satisfies unavailable-type-invariance, weak non-wastefulness, resource-monotonicity, and truncationinvariance, then there exists a priority structure $\succ$ such that the mechanism is $\succ$-stable.

Note that in contrast to Theorem 1 we need truncation-invariance below.

Corollary 4 (Theorem 3 for House Allocation).

In the house allocation model (with a fixed population and variable resources), responsive-deferredacceptance-mechanisms are the only mechanisms satisfying unavailable-type-invariance, weak nonwastefulness, resource-monotonicity, truncation-invariance, and strategy-proofness.

We show the independence of the properties in Corollary 4 in Appendix A.7. Corollaries 3 and 4 were the main results of Ehlers and Klaus (2013).

Next, we briefly discuss two important real-life classes of mechanisms (for house allocation as well as for our more general model) and state their properties.

Remark 2 (The Boston or Immediate-Acceptance-Mechanism).

A mechanism that has also been playing an important role in market design is the so-called Boston or immediate-acceptance mechanism. Any immediate-acceptance mechanism is based on a priority structure and satisfies individual-rationality, weak non-wastefulness, population-monotonicity, and truncation-invariance. However, it is easy to check that the immediate-acceptance mechanism violates strategy-proofness and stability. Whether an immediate-acceptance mechanism satisfies unavailable-type-invariance or resource-monotonicity depends on how available objects are treated in the first round and subsequent rounds of the immediate-acceptance-algorithm:

(i) if agents have to apply to all objects including the ones that are not available, then the resulting immediate-acceptance mechanism satisfies resource-monotonicity (because agents apply to $O \cup\{\emptyset\}$ for any $q$ and at any step of the immediate-acceptance mechanism more objects are available when resources increase) but not unavailable-type-invariance; and

(ii) if agents only apply to objects that are available, then the resulting immediate-acceptance mechanism satisfies unavailable-type-invariance (because agents apply only to $O_{+}(q) \cup\{\emptyset\}$ for any q) but not resource-monotonicity). 
Remark 3 (Top-Trading Cycles Mechanisms).

Another mechanism that has also been playing an important role in market design is the socalled top-trading cycles mechanism (Shapley and Scarf, 1974). Any top-trading cycles (TTC) mechanism is based on a property rights structure (which is given by a priority structure) in order to determine endowments and satisfies unavailable-type-invariance, individual-rationality, weak non-wastefulness, truncation-invariance, and strategy-proofness. However, since priorities can be traded, it is easy to check that any TTC-mechanism violates resource-monotonicity, populationmonotonicity, and stability. Any TTC-mechanism is strategy-proof and efficient. One can see easily that for the house allocation model for a given priority structure, the corresponding TTCmechanism and DA-mechanism coincide if and only if the priority structure is acyclic. In other words, the TTC-mechanism satisfies the comparative statics properties if and only if it is a DAmechanism with an acyclic priority structure.

By Theorem 1 and Corollary 4, in the house allocation model population-monotonicity is a "stronger" comparative statics requirement than resource-monotonicity because (i) populationmonotonicity in conjunction with strategy-proofness and basic properties characterize responsive DA-mechanisms whereas (ii) resource-monotonicity in conjunction with strategy-proofness and basic properties does not characterize responsive DA-mechanisms. On the other hand, (i) populationmonotonicity in conjunction with truncation-invariance and basic properties does not imply stability (Remark 2) whereas (ii) resource-monotonicity in conjunction with truncation-invariance and basic properties implies stability (Corollary 3). We summarize these findings in the Table 1 below (where any mechanism $\varphi$ satisfies our basic properties individual-rationality, weak non-wastefulness, and unavailable-type-invariance).

\begin{tabular}{|l|c|c|}
\hline$\varphi$ is & stable & $\mathrm{DA}$ \\
\hline \hline $\mathrm{PM}$ and TI & no & no \\
$\mathrm{PM}$ and SP & yes & yes \\
$\mathrm{RM}$ & no & no \\
$\mathrm{RM}$ and TI & yes & no \\
$\mathrm{RM}, \mathrm{TI}$, and SP & yes & yes \\
\hline
\end{tabular}

Table 1: RM stands for resource-monotonicity, PM stands for population-monotonicity, TI stands for truncation-invariance, and SP stands for strategy-proofness.

\subsection{Non-Unit Capacities}

In the general model resource-monotonicity is a "stronger" comparative statics requirement than population-monotonicity because (i) resource-monotonicity in conjunction with strategy-proofness, truncation-invariance and basic properties characterizes choice-based DA-mechanisms (Theorem 3) whereas (ii) population-monotonicity in conjunction with strategy-proofness, truncation-invariance and basic properties does not characterize choice-based DA-mechanisms (Example 1). 
We now return to our general allocation model where more than one copy of each real type may be available and the set of agents may vary; a mechanism is a function $\varphi:\left(\cup_{S \in \mathcal{N}} \mathcal{R}^{S}\right) \times \mathcal{Q} \rightarrow \mathcal{A}$.

The following are immediate corollaries from Theorems 2 and 3: if we add populationmonotonicity to the properties in Theorems 2 and 3, then the choice structure will have to be the same across different populations.

Corollary 5 (Existence of a Choice Structure and Stability: Comparative Statics).

If a mechanism $\varphi$ satisfies unavailable-type-invariance, weak non-wastefulness, resourcemonotonicity, population-monotonicity, and truncation-invariance, then there exists a choice structure $\mathcal{C}$ satisfying acceptance, monotonicity, substitutability, and consistency such that $\varphi$ is $\mathcal{C}$-stable.

Corollary 6 (Choice-DA-Mechanisms: Comparative Statics).

Choice-deferred-acceptance-mechanisms (where the choice structure satisfies acceptance, monotonicity, substitutability, and consistency) are the only mechanisms satisfying unavailable-typeinvariance, weak non-wastefulness, resource-monotonicity, population-monotonicity, truncationinvariance, and strategy-proofness.

It is important to notice that neither in Corollary 5 nor in Corollary 6 truncation-invariance can be dropped from the list of properties (even though both comparative statics properties are required). The following example establishes this fact.

Example 2 (Necessity of Truncation-Invariance).

Let $N=\{1,2,3,4\}, O=\{x, y\}$, and there are at most two copies of $x$ and at most one copy of $y$ available, i.e., $\bar{q}_{x}=2$ and $\bar{q}_{y}=1$.

Let $\succ_{x}: 1234, \succ_{x}^{\prime}: 1243$, and $\succ_{y}: 1234$. Let $\succ=\left(\succ_{x}, \succ_{y}\right)$ and $\succ^{\prime}=\left(\succ_{x}^{\prime}, \succ_{y}\right)$. Then, for each $S \in \mathcal{N}$ and each problem $(R, q) \in \mathcal{R}^{S} \times \mathcal{Q}$,

$$
\varphi(R, q)= \begin{cases}D A^{\succ}(R, q) & \text { if } q_{x}=2, q_{y}=1, S=N, \text { and } R_{1}=R_{2}: y x \emptyset \text { and } \\ D A^{\succ^{\prime}}(R, q) & \text { otherwise. }\end{cases}
$$

It is straightforward that $\varphi$ satisfies unavailable-type-invariance, weak non-wastefulness, and strategy-proofness.

First, we show that $\varphi$ violates truncation-invariance. Let $R \in \mathcal{R}^{N}$ be such that $R_{1}=R_{2}: y x \emptyset$, $R_{3}=R_{4}: x \emptyset y$, and $q_{x}=2$ and $q_{y}=1$. Then $\varphi_{3}(R, q)=x$ and $\varphi_{4}(R, q)=\emptyset$. However, for $R_{1}^{\prime} \in \mathcal{R}$ with $R_{1}^{\prime}: y \emptyset x$ and $R^{\prime}=\left(R_{1}^{\prime}, R_{-1}\right)$ we have $\varphi_{4}\left(R^{\prime}, q\right)=x$ and $\varphi_{3}\left(R^{\prime}, q\right)=\emptyset$. Note that $R_{1}^{\prime}$ is a truncation of $R_{1}$ and $\varphi(R, q) \neq \varphi\left(R^{\prime}, q\right)$, i.e., $\varphi$ violates truncation-invariance. Note that this argument also establishes that there exists no acceptant choice structure $\mathcal{C}$ such that $\varphi$ is $\mathcal{C}$-stable: (i) $\varphi_{2}(R, q)=x=\varphi_{3}(R, q)$ and $\varphi_{4}(R, q)=\emptyset$ imply $4 \notin \mathcal{C}_{x}(\{2,3,4\}, 2)$ and (ii) $\varphi_{2}\left(R^{\prime}, q\right)=x=\varphi_{4}\left(R^{\prime}, q\right)$ and $\varphi_{3}\left(R^{\prime}, q\right)=\emptyset$ imply $3 \notin \mathcal{C}_{x}(\{2,3,4\}, 2)$; now (i) and (ii) yield $\left|\mathcal{C}_{x}(\{2,3,4\}, 2)\right|<2$ and $\mathcal{C}_{x}$ is not acceptant. 
Second, in order to see that $\varphi$ satisfies resource-monotonicity, let $R \in \mathcal{R}^{N}$ and $q, q^{\prime} \in \mathcal{Q}$ be such that $q \leq q^{\prime}$ (resource-monotonicity is trivially satisfied when not all agents are present). If $\varphi$ uses the same priority structure for $(R, q)$ and $\left(R, q^{\prime}\right)$, then resource-monotonicity is satisfied. Thus, $q_{y}^{\prime}=1, q_{x}^{\prime}=2$, and $R_{1}=R_{2}: y x \emptyset$. Note that the difference in $\succ_{x}$ and $\succ_{x}^{\prime}$ only matters for $R \in \mathcal{R}^{N}$ with $x \in A\left(R_{3}\right) \cap A\left(R_{4}\right)$. Then, for all the following specifications of $q$ and $q^{\prime}$ we see that resource-monotonicity is satisfied (see Table 2 below).

\begin{tabular}{|c|l|l|}
\hline$q=\left(q_{y}, q_{x}\right)$ & $\varphi(R, q)$ & $\varphi\left(R, q^{\prime}\right)$ \\
\hline \hline$(0,0)$ & $(\emptyset, \emptyset, \emptyset, \emptyset)$ & $(y, x, x, \emptyset)$ \\
$(1,0)$ & $(y, \emptyset, \emptyset, \emptyset))$ & $(y, x, x, \emptyset)$ \\
$(0,1)$ & $(x, \emptyset, \emptyset, \emptyset)$ & $(y, x, x, \emptyset)$ \\
$(1,1)$ & $(y, x, \emptyset, \emptyset)$ & $(y, x, x, \emptyset)$ \\
$(0,2)$ & $(x, x, \emptyset, \emptyset)$ & $(y, x, x, \emptyset)$ \\
\hline
\end{tabular}

Table 2: Resource-Monotonicity is satisfied.

Third, in order to see that $\varphi$ satisfies population-monotonicity, let $(R, q) \in \mathcal{R}^{N} \times \mathcal{Q}$ and $S \subseteq N$ with $|S|=2,3$. If $\varphi$ uses the same priority structure for $(R, q)$ and $\left(R_{S}, q\right)$, then populationmonotonicity is satisfied. Thus, $q_{y}=1, q_{x}=2$, and $R_{1}=R_{2}: y x \emptyset$. Furthermore (for different priorities) to be employed, we must have $3,4 \in S$ and $S \in\{\{1,3,4\},\{2,3,4\},\{3,4\}\}$.

For $S=\{3,4\}, D A^{\succ}\left(R_{S}, q\right)=D A^{\succ^{\prime}}\left(R_{S}, q\right)$ (because $\succ_{y}=\succ_{y}^{\prime}$ and two copies of $x$ are available). For $S=\{i, 3,4\}, i \in\{1,2\}, D A_{i}^{\succ}\left(R_{S}, q\right)=D A_{i}^{\succ^{\prime}}\left(R_{S}, q\right)=y$, and $D A_{3}^{\succ}\left(R_{S}, q\right)=D A_{3}^{\succ^{\prime}}\left(R_{S}, q\right)$, and $D A_{4}^{\succ}\left(R_{S}, q\right)=D A_{4}^{\succ^{\prime}}\left(R_{S}, q\right)$ (because there are two copies of $x$ for agents 3 and 4 ). Since the use of the two possible different priorities is outcome equivalent, population-monotonicity is satisfied. $\diamond$

Next, we present an example of an acceptant and substitutable choice structure that is not responsive.

Example 3 (Non-Responsiveness of a Substitutable Choice Structure).

Let $N=\left\{s_{1}, s_{2}, j_{1}, j_{2}\right\}$ be four economists looking for a new academic position. Furthermore, $s_{1}, s_{2}$ are seniors with specializations 1 or 2 and $j_{1}, j_{2}$ are juniors with specializations 1 or 2 . The intuition behind the priorities that we define is the following. An economics department $x \in O$ has the following preferences for hiring. If only one economist can be hired the priority ranking for hiring is $s_{1} \succ_{x} s_{2} \succ_{x} j_{1} \succ_{x} j_{2}$. However, if two positions can be filled, the department always would like to fill both positions. So, $\bar{q}_{x}=2$. Furthermore, they would like to hire the two seniors; but if only one senior $s_{i}$ can be hired, then they are interested in also hiring the junior $j_{i}$ in the same field. To be more specific, we assume that the department's priority ranking for hiring two economists is $\left\{s_{1}, s_{2}\right\} \succ_{x}\left\{s_{1}, j_{1}\right\} \succ_{x}\left\{s_{2}, j_{2}\right\} \succ_{x}\left\{s_{1}, j_{2}\right\} \succ_{x}\left\{s_{2}, j_{1}\right\} \succ_{x}\left\{j_{1}, j_{2}\right\}$.

Priorities $\mathcal{C}_{x}$ based on these preferences work as follows. For $q_{x}=2$ (the case $\mathcal{C}_{x}(M, 1)$ is obvious) it follows that 


$$
\mathcal{C}_{x}(M, 2)=\left\{\begin{array}{cl}
M & \text { if }|M| \leq 2, \\
\left\{s_{1}, s_{2}\right\} & \text { if }\left\{s_{1}, s_{2}\right\} \varsubsetneqq M, \\
\left\{s_{i}, j_{i}\right\} & \text { if }\left\{s_{i}, j_{i}\right\} \varsubsetneqq M, i \in\{1,2\} .
\end{array}\right.
$$

It is easily verified that $\mathcal{C}_{x}$ is an acceptant, substitutable, and consistent choice function. Since $\mathcal{C}_{x}\left(\left\{s_{1}, j_{1}, j_{2}\right\}, 2\right)=\left\{s_{1}, j_{1}\right\}$ and $\mathcal{C}\left(\left\{s_{2}, j_{1}, j_{2}\right\}, 2\right)=\left\{s_{2}, j_{2}\right\}, \mathcal{C}_{x}$ is not responsive (because responsiveness would imply $\left.j_{1} \succ_{x} j_{2} \Leftrightarrow\left\{s_{2}, j_{1}\right\} \succ_{x}\left\{s_{2}, j_{2}\right\}\right)$.

\subsection{Deferred-Acceptance Characterizations: Connections with Previous Papers}

Kojima and Manea (2010) were the first to obtain characterizations of choice-deferred-acceptancemechanisms for a fixed-population, fixed-resource, and general capacity model. Ehlers and Klaus (2014) characterize the smaller class of responsive-deferred-acceptance-mechanisms for a variable-resource and general capacity model (in one of their characterizations the population is fixed, in the other characterization the population varies). Next, we discuss the relation of our main result for the fixed-population, variable-resource, and general capacity model, Theorem 3, with the main results in Kojima and Manea (2010, Theorems 1 and 2) and Ehlers and Klaus (2014, Theorems 1 and 4).

Note that in all these contributions, priorities are derived from a mechanism via a set of properties. Other papers take exogenous priorities as given and impose properties on the mechanism using these exogenous priorities. Morrill (2013, Theorems 1 and 2) is an example of this approach, but since his results are closely related to the results of Kojima and Manea (2010, Theorems 1 and 2 ), we add them to our review of related results.

\subsubsection{Ehlers and Klaus (2014)}

For a variable-resource and general capacity model, Ehlers and Klaus (2014, Theorems 1 and 4) provide two characterizations of responsive-deferred-acceptance-mechanisms (where the choice structure satisfies acceptance, substitutability, and consistency).

We denote a preference relation with only one acceptable type $x \in O$ by $R^{x}$, i.e., $A\left(R^{x}\right)=\{x\}$. We denote the set of all preference relations that have $x \in O$ as the unique acceptable type by $\mathcal{R}^{x}$. A requirement which played a key role in Ehlers and Klaus (2014, Theorem 1) for a fixed population $N$ is the following.

Two-Agent-Consistent-Conflict-Resolution: For all $R \in \mathcal{R}^{N}$, all $q, q^{\prime} \in \mathcal{Q}$, and all $R^{x} \in \mathcal{R}^{x}$, if $R_{i}=R_{j}=R^{x}$ and $\left\{\varphi_{i}(R, q), \varphi_{j}(R, q)\right\}=\left\{\varphi_{i}\left(R, q^{\prime}\right), \varphi_{j}\left(R, q^{\prime}\right)\right\}=\{x, \emptyset\}$, then for $k \in\{i, j\}$, $\varphi_{k}(R, q)=\varphi_{k}\left(R, q^{\prime}\right)$.

One can interpret this property as a weak tie-breaking property because it only imposes that the tie between two agents in very specific maximal conflict situations is broken consistently in 
favor of the same agent. It is obvious that in Example 3, the induced choice-based DA-mechanism violates this property (because the choice structure is not responsive).

For a fixed-population, variable-resource, and general capacity model, Ehlers and Klaus (2014, Theorem 1) prove that responsive-deferred-acceptance-mechanisms are the only mechanisms satisfying unavailable-type-invariance, individual-rationality, weak non-wastefulness, two-agentconsistent-conflict-resolution, truncation-invariance, and strategy-proofness. Hence, with Theorem 3 we demonstrate that by replacing two-agent-consistent-conflict-resolution by resourcemonotonicity, we obtain a larger class of mechanisms. It is also interesting to note that while two-agent-consistent-conflict-resolution is a property that is very specific to private and discrete goods allocation situations, resource-monotonicity is a very intuitive and general property that applies to a wide range of private as well as public goods allocation situations.

Two requirements that played a key role in Ehlers and Klaus (2014, Theorem 4) are unassignedtype-invariance and weak consistency.

Unassigned-type-invariance is very much in the spirit of unavailable-type-invariance: the chosen allocation does not depend on the unconsumed or unassigned objects. For all $S \in \mathcal{N}$ and all $(R, q) \in \mathcal{R}^{S} \times \mathcal{Q}$, we define by $q(\varphi(R, q))$ the capacity vector of assigned objects: for all $x \in O$, $q_{x}(\varphi(R, q))=\left|\left\{j \in S: \varphi_{j}(R, q)=x\right\}\right|$.

Unassigned Type Invariance: For all $S \in \mathcal{N}$ and all $(R, q) \in \mathcal{R}^{S} \times \mathcal{Q}, \varphi(R, q)=\varphi(R, q(\varphi(R, q)))$.

Weak consistency requires that if some agents leave a problem with their allotments, then an agent who did not leave and who received the null object, still receives the null object.

Weak Consistency: For all $T, S \in \mathcal{N}$ such that $T \subseteq S$, all $R \in \mathcal{R}^{S}$, all $q \in \mathcal{Q}$, and all $i \in T$, if $\varphi_{i}(R, q)=\emptyset$, then $\varphi_{i}\left(R_{T}, \tilde{q}\right)=\emptyset$ where $\tilde{q}_{x}=q_{x}-\left|\left\{j \in S \backslash T: \varphi_{j}(R, q)=x\right\}\right|$ for all $x \in O$.

For a variable-population, variable-resource, and general capacity model, Ehlers and Klaus (2014, Theorem 4) prove that responsive-deferred-acceptance-mechanisms are the only mechanisms satisfying unassigned-type-invariance, individual-rationality, weak non-wastefulness, weak consistency, and strategy-proofness.

\subsubsection{Kojima and Manea (2010) and Morrill (2013)}

For a fixed-population and fixed-resource general capacity model, Kojima and Manea (2010, Theorems 1 and 2) provide two characterizations of choice-deferred-acceptance-mechanisms (where the choice structure satisfies acceptance, substitutability, and consistency). Non-wastefulness as used by Kojima and Manea (2010) and Morrill (2013) $)^{9}$ is equivalent to individual rationality and our non-wastefulness. In the characterizations obtained by Kojima and Manea (2010) and by

\footnotetext{
${ }^{9}$ K-M-Non-Wastefulness: For all $(R, q) \in \mathcal{R}^{N} \times \mathcal{Q}$, all $x \in O_{+}(q) \cup\{\emptyset\}$ with $\left|\left\{j \in N: \varphi_{j}(R, q)=x\right\}\right|<q_{x}$, and all $i \in N, \varphi_{i}(R, q) R_{i} x$.
} 
Morrill (2013), next to non-wastefulness, two monotonicity properties are employed: individuallyrational-monotonicity and weak Maskin-monotonicity.

Given $i \in N$ and $R_{i} \in \mathcal{R}$, a strategy $\bar{R}_{i} \in \mathcal{R}$ is an individually-rational-monotonic transformation of $R_{i}$ at $\varphi_{i}(R, q)$ if for all $x \in O, x \bar{P}_{i} \varphi_{i}(R, q)$ and $x \bar{P}_{i} \emptyset$ imply $x P_{i} \varphi_{i}(R, q)$.

Individually-Rational-Monotonicity: For all $(R, q) \in \mathcal{R}^{N} \times \mathcal{Q}$, all $i \in N$, and all $\bar{R}_{i} \in \mathcal{R}_{i}$, if $\bar{R}_{i}$ is an individually-rational-monotonic transformation of $R_{i}$ at $\varphi_{i}(R, q)$ and $\bar{R}=\left(\bar{R}_{i}, R_{-i}\right)$, then for all $j \in N, \varphi_{j}(\bar{R}, q) \bar{R}_{j} \varphi_{j}(R, q)$.

Given $i \in N$ and $R_{i} \in \mathcal{R}$, a strategy $\bar{R}_{i} \in \mathcal{R}$ is a monotonic transformation of $R_{i}$ at $\varphi_{i}(R, q)$ if for all $x \in O \cup\{\emptyset\}, x \bar{P}_{i} \varphi_{i}(R, q)$ implies $x P_{i} \varphi_{i}(R, q)$.

Weak Maskin-Monotonicity: For all $(R, q) \in \mathcal{R}^{N} \times \mathcal{Q}$, all $i \in N$, and all $\bar{R}_{i} \in \mathcal{R}_{i}$, if $\bar{R}_{i}$ is a Maskin monotonic transformation of $R_{i}$ at $\varphi_{i}(R, q)$ and $\bar{R}=\left(\bar{R}_{i}, R_{-i}\right)$, then for all $j \in N$, $\varphi_{j}(\bar{R}, q) \bar{R}_{j} \varphi_{j}(R, q)$.

It can be shown that either of the two monotonicity properties implies truncation-invariance.

For a fixed-population, fixed-resource, and general capacity model, Kojima and Manea (2010) characterize choice-deferred-acceptance-mechanisms (where the choice structure satisfies acceptance, substitutability, and consistency) either by non-wastefulness (and individual-rationality) and individually-rational-monotonicity (Kojima and Manea, 2010, Theorem 1) or by non-wastefulness (and individual-rationality), population-monotonicity (for fixed-populations), and weak Maskinmonotonicity (Kojima and Manea, 2010, Theorem 2).

Morrill (2013, Theorems 1 and 2) builds on the results by Kojima and Manea (2010) and provides characterizations of choice-deferred-acceptance-mechanisms that are defined with any given substitutable choice structure as additional input. This is achieved by only adding the very weak stability condition of mutually-best that requires that whenever an agent has the highest priority at his favorite type, it is assigned to the agent.

Mutually Best: For all $S \in \mathcal{N}$ and all $(R, q) \in \mathcal{R}^{S} \times \mathcal{Q}$, if $i \in S$ and $x \in O$ are such that for all $y \in O \backslash\{x\} \cup\{\emptyset\}, x P_{i} y$ and $i \in \mathcal{C}_{x}\left(S, q_{x}\right)$, then $\varphi_{i}(R, q)=x$.

For a fixed-population, fixed-resource, and general capacity model, Morrill (2013) characterizes choice-deferred-acceptance-mechanisms (where the choice structure is given and satisfies acceptance, substitutability, and consistency) either by non-wastefulness (and individual-rationality), individually-rational-monotonicity, and mutually-best (Morrill, 2013, Theorem 2) or by nonwastefulness (and individual-rationality), population-monotonicity (for fixed-populations), weak Maskin-monotonicity, and mutually-best (Morrill, 2013, Theorem 1). 


\subsubsection{Characterizations of Deferred-Acceptance}

The following Table 3 summarizes the discussed characterizations of the agent-proposing deferredacceptance mechanisms for the general capacity model.

\begin{tabular}{|l||c|c|c|c|c|c|c|c|c|c|c|c|l||l|}
\hline Unit & UTI & IR & NW & WNW & PM & RM & TI & SP & 2C & WC & IRM & WM & MB & Non-unit \\
\hline \hline Th. 1: R-DA & & + & & + & + & & & + & & & & & & Ex. 1: No \\
\hline Yes & + & & & + & & + & + & + & & & & & & Th. 3: C-DA \\
\hline Yes & + & & & + & + & + & + & + & & & & & & Co. 6: C-DA \\
\hline Yes & + & + & & + & & & + & + & + & & & & & Th. 1 (EK): R-DA \\
\hline Yes & + & + & & + & & & & + & & + & & & & Th. 4 (EK): R-DA \\
\hline Yes & & + & + & & & & & & & & + & & & Th. 1 (KM): C-DA \\
\hline Yes & & + & + & & & & & & & & + & & + & Th. 2 (MO): C $^{*}$-DA \\
\hline Yes & & + & + & & + & & & & & & & + & & Th. 2 (KM): C-DA \\
\hline Yes & & + & + & & + & & & & & & & + & + & Th. 1 (MO): C*-DA \\
\hline
\end{tabular}

Table 3: The most left column stands for unit capacities and the most right column for non-unit capacities. In the most left column we only write "Yes" when the characterization holds for general capacities (and unit capacities is a special case of the characterization for non-unit capacities).

UTI stands for unavailable-type-invariance,

IR stands for individual-rationality,

WNW stands for weak non-wastefulness,

2C stands for two-agent-consistent-conflict-resolution,

WC stands for weak consistency,

IRM stands for individually-rational-monotonicity,

WM stands for weak Maskin-monotonicity,

MB stand for mutually-best.

R-DA stands for responsive-DA-mechanisms,

C-DA stands for choice-DA-mechanisms,

$\mathrm{C}^{*}$-DA stands for choice-DA-mechanisms with given choice structures.

EK refers to Ehlers and Klaus (2014),

KM refers to Kojima and Manea (2010),

MO refers to Morrill (2013).

Furthermore, in the line corresponding to Th.4 (EK), the "+" for UTI stands implicitly for unassigned-type-invariance instead of unavailable-type-invariance. 


\section{References}

Arrow, K.J. (1951): Social Choice and Individual Values. New York: Wiley.

Aygün, O., and T. Sönmez (2013): Matching with Contracts: Comment, American Economic Review 103, 2050-2051.

Balinski, M., and T. Sönmez (1999): A Tale of Two Mechanisms: Student Placement, Journal of Economic Theory 84, 73-94.

Chambers, C.P., and M.B. Yenmez (2014): Choice and Matching, Working Paper.

Chun, Y., and W. Thomson (1988): Monotonicity Properties of Bargaining Solutions when Applied to Economics, Mathematical Social Sciences 15, 11-27.

Crawford, V.P. (1991): Comparative Statics in Matching Markets, Journal of Economic Theory $54,389-400$.

Dubins, L.E., and D.A. Freedman (1981): Machiavelli and the Gale-Shapley Algorithm, American Mathematical Monthly 88, 485-494.

Ehlers, L. (2002): Coalitional Strategy-Proof House Allocation, Journal of Economic Theory 105, $298-317$.

Ehlers, L., I.E. Hafalir, M.B. Yenmez, and M.A. Yildirim (2014): School Choice with Controlled Choice Constraints: Hard Bounds vs. Soft Bounds, Journal of Economic Theory 153, 648-68.

Ehlers, L., and B. Klaus (2004): Resource-Monotonic House Allocation, International Journal of Game Theory 32, 545-560.

Ehlers, L., and B. Klaus (2007): Consistent House Allocation, Economic Theory 30, 561-574.

Ehlers, L., and B. Klaus (2011): Corrigendum to "Resource-Monotonicity for House Allocation Problems", International Journal of Game Theory 40, 281-287.

Ehlers. L., and B. Klaus (2013): House Allocation via Deferred-Acceptance, Cahier de recherches économiques du DEEP No. 13.10.

Ehlers, L., and B. Klaus (2014): Strategy-Proofness makes the Difference: Deferred-Acceptance with Responsive Priorities, Mathematics of Operations Research 39, 949-966.

Ehlers, L., B. Klaus, and S. Pápai (2002): Strategy-Proofness and Population-Monotonicity for House Allocation Problems, Journal of Mathematical Economics 38, 329-339.

Ergin, H.İ. (2002): Efficient Resource Allocation on the Basis of Priorities, Econometrica 70, 2489-2497.

Fleurbaey, M., and K. Tadenuma (2007): Do Irrelevant Commodities Matter?, Econometrica 75, $1143-1174$.

Gale, D., and L. Shapley (1962): College Admissions and the Stability of Marriage, American Mathematical Monthly 69, 9-15. 
Hatfield, J.W., and P.R. Milgrom (2005): Matching with Contracts, American Economic Review 95, 913-935.

Kamada, Y., and F. Kojima (2015): General Theory of Matching under Distributional Constraints, Working Paper.

Kelso, A.S., and V.P. Crawford (1982): Job Matching, Coalition Formation, and Gross Substitutes, Econometrica 6, 1483-1504.

Kesten, O. (2009): Coalitional Strategy-Proofness and Resource Monotonicity for House Allocation Problems, International Journal of Game Theory 38, 17-21.

Kojima, F., and M. Manea (2010): Axioms for Deferred Acceptance, Econometrica 78, 633-653.

Kumano, T. (2009): Efficient Resource Allocation under Acceptant Substitutable Priorities, Working Paper.

Morrill, T. (2013): An Alternative Characterization of the Deferred Acceptance Algorithm, International Journal of Game Theory 42, 19-28.

Pápai, S. (2000): Strategyproof Assignment by Hierarchical Exchange, Econometrica 68, 14031433.

Roth, A.E. (1982): The Economics of Matching: Stability and Incentives, Mathematics of Operations Research 7, 617-628.

Roth, A.E. (1984): Stability and Polarization of Interests in Job Matching, Econometrica 52, $47-57$.

Roth, A.E., and M.A.O. Sotomayor (1990): Two-Sided Matching: A Study in Game-Theoretic Modeling and Analysis. Econometric Society Monograph Series. New York: Cambridge University Press.

Shapley, L., and H. Scarf (1974): On Cores and Indivisibility, Journal of Mathematical Economics $1,23-37$.

Thomson, W. (1983): The Fair Division of a Fixed Supply Among a Growing Population, Mathematics of Operations Research 8, 319-326.

Thomson, W. (1995): Population Monotonic Allocation Rules, in Social Choice, Welfare and Ethics, eds. W.A. Barnett, H. Moulin, M. Salles, and N. Schofield, Cambridge University Press. 


\section{A Appendix}

\section{A.1 Proof of Theorem 1}

Recall that in the house allocation model at most one object per real type is available. It therefore suffices to denote a problem by $R \in \mathcal{R}^{S}(S \in \mathcal{N})$. Also recall that we consider variable-population mechanisms $\varphi:\left(\cup_{S \in \mathcal{N}} \mathcal{R}^{S}\right) \rightarrow \mathcal{A}$ (with a fixed set of types $O$ ).

First, note that all responsive-deferred-acceptance mechanisms are stable and that stability implies individual-rationality and weak non-wastefulness. Dubins and Freedman (1981) and Roth (1982) proved strategy-proofness of all responsive-deferred-acceptance-mechanisms. Crawford (1991) studied comparative statics of deferred-acceptance-mechanisms (for a more general model than that of housing markets). From his results it follows that all responsive-deferred-acceptance-mechanisms are population-monotonic. Hence, responsive-deferredacceptance-mechanisms satisfy all properties of Theorem 1.

Second, let $\varphi$ be a mechanism satisfying individual-rationality, weak non-wastefulness, and population-monotonicity (we will add strategy-proofness later on). We construct a priority structure using maximal conflict preference profiles.

Let $x \in O$ and $R^{x} \in \mathcal{R}$ be such that $A\left(R^{x}\right)=\{x\}$. We denote the set of all preference relations that have $x \in O$ as the unique acceptable type by $\mathcal{R}^{x}$. For any $S \in \mathcal{N}$, let $R_{S}^{x}=\left(R_{i}^{x}\right)_{i \in S}$ such that for all $i \in S, R_{i}^{x}=R^{x}$.

Consider the problem $R_{N}^{x}$. By weak non-wastefulness and individual-rationality, for some $j \in$ $N, \varphi_{j}\left(R_{N}^{x}\right)=x$, say $j=1$. Then, for all $i \in N \backslash\{1\}$, we set $1 \succ_{x} i$.

Next consider the problem $R_{N \backslash\{1\}}^{x}$. By weak non-wastefulness and individual-rationality, for some $j \in N \backslash\{1\}, \varphi_{j}\left(R_{N \backslash\{1\}}^{x}\right)=x$, say $j=2$. Then, for all $i \in N \backslash\{1,2\}$, we set $2 \succ_{x} i$.

By induction, we obtain $\succ_{x}$ for any real type $x$ and thus a priority structure $\succ=\left(\succ_{x}\right)_{x \in O}$ for $N$. By weak non-wastefulness, individual-rationality, and population-monotonicity, for any $x \in O$ and any $i, j \in N$ such that $i \succ_{x} j, \varphi_{i}\left(R^{x}, R^{x}\right)=x$ and $\varphi_{j}\left(R^{x}, R^{x}\right)=\emptyset$.

Let mechanism $\varphi$ satisfy strategy-proofness. With the following step, Theorem 1 follows. Note that mechanism $D A^{\succ}$ satisfies all properties of Theorem 1.

Let $S \in \mathcal{N}, R \in \mathcal{R}^{S}$, and denote by $Z(R):=\left|\left\{i \in S:\left|A\left(R_{i}\right)\right| \leq 1\right\}\right|$ the number of agents who find at most one object acceptable. Assume that $R \in \mathcal{R}^{S}$ is such that $\varphi(R) \neq D A^{\succ}(R)$ and that $Z(R)$ is maximal, i.e., for all $R^{\prime} \in \mathcal{R}^{S}$ such that $\varphi\left(R^{\prime}\right) \neq D A^{\succ}\left(R^{\prime}\right), Z(R) \geq Z\left(R^{\prime}\right)$.

We first show that for all $i \in S$ such that $\varphi_{i}(R) \neq D A_{i}^{\succ}(R),\left|A\left(R_{i}\right)\right|=1$. If $\varphi_{i}(R) P_{i} D A_{i}^{\succ}(R)$, by individual-rationality, $\varphi_{i}(R):=x \in O$. If $\left|A\left(R_{i}\right)\right|=1$, then we are done. If $\left|A\left(R_{i}\right)\right|>1$, then consider $\left(R^{x}, R_{-i}\right) \in \mathcal{R}^{S}$ where $R^{x}$ as in the construction of $\succ$. By strategy-proofness (and individual-rationality), $\varphi_{i}\left(R^{x}, R_{-i}\right)=x$ and $D A_{i}^{\succ}\left(R^{x}, R_{-i}\right)=\emptyset$. Hence, $\left(R^{x}, R_{-i}\right)$ is such that $\varphi\left(R^{x}, R_{-i}\right) \neq D A^{\succ}\left(R^{x}, R_{-i}\right)$ and $Z\left(R^{x}, R_{-i}\right)>Z(R)$; contradicting our assumption that $Z(R)$ was maximal. If $D A_{i}^{\succ}(R) P_{i} \varphi_{i}(R)$, then $D A_{i}^{\succ}(R):=x \in O$ and the proof that $\left|A\left(R_{i}\right)\right|=1$ 
proceeds as before (with $D A^{\succ}$ in the role of $\varphi$ and vice versa). Hence, for all $i \in S$ such that $\varphi_{i}(R) \neq D A_{i}^{\succ}(R),\left|A\left(R_{i}\right)\right|=1$. By strategy-proofness, we can assume that for all $i \in S$ such that $\varphi_{i}(R) \neq D A_{i}^{\succ}(R), R_{i}=R^{x}$ where $R^{x}$ is as in the construction of $\succ$.

If $x=D A_{i}^{\succ}(R) P_{i} \varphi_{i}(R)=\emptyset$, then by weak non-wastefulness there exists $j \in S \backslash\{i\}$ such that $\varphi_{j}(R)=x$. Hence, $\varphi_{j}(R) \neq D A_{j}^{\succ}(R)$ and $R_{j}=R^{x}$ where $R^{x}$ is as in the construction of $\succ$. Thus, $\left(R_{i}, R_{j}\right)=\left(R^{x}, R^{x}\right)$ and $x=\varphi_{j}(R) P_{j} D A_{j}^{\succ}(R)=\emptyset$. Now $D A_{i}^{\succ}(R)=x$ and populationmonotonicity (and individual-rationality) for $D A^{\succ}$ imply $D A_{i}^{\succ}\left(R_{i}, R_{j}\right)=x$ and $D A_{j}^{\succ}\left(R_{i}, R_{j}\right)=\emptyset$. Hence, $i \succ_{x} j$. Next, $\varphi_{j}(R)=x$ and population-monotonicity (and individual-rationality) for $\varphi$ imply $\varphi_{j}\left(R_{i}, R_{j}\right)=x$ and $\varphi_{i}\left(R_{i}, R_{j}\right)=\emptyset$. Hence, $j \succ_{x} i$; a contradiction.

If $x=\varphi_{i}(R) P_{i} D A_{i}^{\succ}(R)=\emptyset$, then by weak non-wastefulness there exists $j \in S \backslash\{i\}$ such that $D A_{j}^{\succ}(R)=x$. Hence, we obtain a contradiction as in the previous step.

\section{A.2 Independence of Properties in Theorem 1}

Recall that we denote a problem by $R \in \mathcal{R}^{S}(S \in \mathcal{N})$ and that we consider variable-population mechanisms $\varphi:\left(\cup_{S \in \mathcal{N}} \mathcal{R}^{S}\right) \rightarrow \mathcal{A}$ (with a fixed set of types $O$ ). Without loss of generality we assume $N=\{1, \ldots, n\}$ to establish independence.

For any strict order $\pi$ of agents in $N$, we denote the corresponding serial dictatorship mechanism by $f^{\pi}$; for example, if $\pi: 12 \ldots(n-1) n$, then $f^{\pi}$ works as follows: for each problem $R \in \mathcal{R}^{S}$ $(S \in \mathcal{N})$, first agent $\min S$ chooses his preferred object in $O \cup\{\emptyset\}$, then agent $\min (S \backslash\{\min S\})$ chooses his preferred object from the remaining objects $\left(O \backslash\left\{f_{\min S}^{\pi}(R)\right\}\right) \cup\{\emptyset\}$, etc. Note that for each strict order $\pi$ of $N, f^{\pi}=D A^{\succ^{\pi}}$ where $\succ^{\pi}$ equals the strict order $\pi$, i.e., for all $x \in O, \succ_{x}^{\pi}=\pi$. Thus, each serial dictatorship mechanism $f^{\pi}$ satisfies individual-rationality, weak non-wastefulness, population-monotonicity, truncation-invariance, and strategy-proofness.

The following examples establish the independence of the properties (properties not mentioned in the examples follow easily) in Theorem 1.

Not individually-rational: Let $\pi: 12 \ldots(n-1) n$ and $|O| \geq|N|$. For each $R_{n} \in \mathcal{R}$, let $\hat{R}_{n}$ be such that $A\left(\hat{R}_{n}\right)=O$ and $\hat{R}_{n}\left|O=R_{n}\right| O$. Then, for each $R \in \mathcal{R}^{S}(S \in \mathcal{N})$,

$$
\varphi(R)= \begin{cases}f^{\pi}\left(R_{-n}, \hat{R}_{n}\right) & \text { if } n \in S \text { and } \\ f^{\pi}(R) & \text { otherwise. }\end{cases}
$$

Note that under $f$ agent $n$ always is assigned a real object because $|O| \geq|N|$.

Not weakly non-wasteful: Fix a type $y \in O$ and $\pi: 12 \ldots(n-1) n$. Then, for each $R \in \mathcal{R}^{S}$ $(S \in \mathcal{N})$, let $\hat{R}^{y} \in \mathcal{R}^{S}$ be such that $\hat{R}^{y}|(O \backslash\{y\}) \cup\{\emptyset\}=R|(O \backslash\{y\}) \cup\{\emptyset\}, A\left(\hat{R}_{i}^{y}\right)=A\left(R_{i}\right) \backslash\{y\}$, and

$$
\varphi(R)=f^{\pi}\left(\hat{R}^{y}\right)
$$


Not population-monotonic: ${ }^{10}$ Let $\pi$ and $\pi^{\prime}$ be two distinct strict orders of agents in $N$. Then, for each $R \in \mathcal{R}^{S}(S \in \mathcal{N})$,

$$
\varphi(R)= \begin{cases}f^{\pi}(R) & \text { if } S=N \text { and } \\ f^{\pi^{\prime}}(R) & \text { otherwise. }\end{cases}
$$

Not strategy-proof: Let $\succ$ be a priority structure. Then, the responsive-deferred-acceptancemechanism based on the type-optimal stable allocation that is obtained by using Gale and Shapley's (1962) type-proposing deferred-acceptance-algorithm satisfies all properties except strategyproofness.

\section{A.3 Proof of Corollary 1}

Acyclic responsive-deferred-acceptance mechanisms satisfy all the properties listed in the corollary.

(a) Let $\varphi$ be a mechanism satisfying efficiency, population-monotonicity, and strategy-proofness.

Efficiency implies individual-rationality and weak non-wastefulness. Then, by Theorem 1, there exists a priority structure $\succ$ such that $\varphi=D A^{\succ}$. Finally, by efficiency and Ergin (2002, Theorem 1), $\succ$ must be acyclic.

(b) Let $\varphi$ be a mechanism satisfying individual-rationality, weak non-wastefulness, populationmonotonicity, and group-strategy-proofness.

Group-strategy-proofness implies strategy-proofness. Hence, by Theorem 1, there exists a priority structure $\succ$ such that $\varphi=D A^{\succ}$. Finally, by group-strategy-proofness and Ergin (2002, Theorem 1), $\succ$ must be acyclic.

\section{A.4 Proof of Theorems 2 and 3}

Let $\mathcal{C}=\left(\mathcal{C}_{x}\right)_{x \in O}$ be an acceptant, monotonic, substitutable, and consistent choice structure. First, note that the corresponding choice-deferred-acceptance-mechanism is $\mathcal{C}$-stable and that $\mathcal{C}$-stability implies weak non-wastefulness. Furthermore, it is easy to check that $D A^{\mathcal{C}}$ satisfies unavailabletype-invariance and truncation-invariance. Hatfield and Milgrom (2005) proved strategy-proofness of the choice-deferred-acceptance-mechanisms based on an acceptant, substitutable, and consistent choice structure $\mathcal{C}$. The most general result implying resource-monotonicity (and populationmonotonicity) for choice-deferred-acceptance-mechanism based on substitutable, and consistent choice structures $\mathcal{C}$ we are aware of is Chambers and Yenmez (2014, Corollary 1). ${ }^{11}$ Hence, choicedeferred-acceptance-mechanisms that are based on acceptant, monotonic, substitutable, and consistent choice structures satisfy all properties of Theorem 3 (and hence of Theorem 2).

\footnotetext{
${ }^{10}$ Again note that here we could have chosen the TTC-mechanism as an example satisfying all properties in Theorem 1 except for population-monotonicity.

${ }^{11}$ Chambers and Yenmez (2014, Theorem 2) extend the previous comparative statics results obtained by Crawford (1991, Theorems 1 and 2).
} 
Second, let $\varphi$ be a mechanism satisfying unavailable-type-invariance, weak non-wastefulness, resource-monotonicity, and truncation-invariance (we will first complete the proof of Theorem 2 and then add strategy-proofness later on to complete the proof of Theorem 3). Note that by Remark 1, resource-monotonicity implies individual-rationality. We construct a choice structure by using maximal conflict preference profiles.

For any $x \in O$ let $R^{x} \in \mathcal{R}$ be such that $A\left(R^{x}\right)=\{x\}$. For any $S \in \mathcal{N}$, let $R_{S}^{x}=\left(R_{i}^{x}\right)_{i \in S}$ such that for all $i \in S, R_{i}^{x}=R^{x}$. Given $l \in\left\{1, \ldots, \bar{q}_{x}\right\}$, let $l_{x}$ denote the capacity vector where $l$ copies of $x$ are available and no copy of any other real object is available. Let $R^{\emptyset} \in \mathcal{R}$ be such that $A\left(R^{\emptyset}\right)=\emptyset$. For any $S \in \mathcal{N}$, let $R_{S}^{\emptyset}=\left(R_{i}^{\emptyset}\right)_{i \in S}$ such that for all $i \in S, R_{i}^{\emptyset}=R^{\emptyset}$.

For any $x \in O$, let $\mathcal{C}_{x}: \mathcal{N} \times\left\{1, \ldots, \bar{q}_{x}\right\} \rightarrow \mathcal{N}$ be such that for all $S \in \mathcal{N}$ and $l \in\left\{1, \ldots, \bar{q}_{x}\right\}$, $\mathcal{C}_{x}(S, l)=\left\{i \in S: \varphi_{i}\left(R_{S}^{x}, R_{N \backslash S}^{\emptyset}, l_{x}\right)=x\right\}$ denotes the choice made at the maximal conflict problem $\left(R_{S}^{x}, l_{x}\right)$. By weak non-wastefulness, $\mathcal{C}_{x}$ is acceptant, i.e., $\left|\mathcal{C}_{x}(S, l)\right|=\min \{|S|, l\}$.

Lemma 2. For all $x \in O, \mathcal{C}_{x}$ is substitutable.

Proof. Let $x \in O$. Let $s, s^{\prime} \in S$ with $s \neq s^{\prime}$ and $l \in\left\{1, \ldots, \bar{q}_{x}\right\}$. To show substitutability of $\mathcal{C}_{x}$ we need to show that $s \in \mathcal{C}_{x}(S, l)$ implies $s \in \mathcal{C}_{x}\left(S \backslash\left\{s^{\prime}\right\}, l\right)$. By definition, $\mathcal{C}_{x}(S, l)=\{i \in S$ : $\left.\varphi_{i}\left(R_{S}^{x}, R_{N \backslash S}^{\emptyset}, l_{x}\right)=x\right\}$ and $\mathcal{C}_{x}\left(S \backslash\left\{s^{\prime}\right\}, l\right)=\left\{i \in S \backslash\left\{s^{\prime}\right\}: \varphi_{i}\left(R_{S \backslash\left\{s^{\prime}\right\}}^{x}, R_{(N \backslash S) \cup\left\{s^{\prime}\right\}}^{\emptyset}, l_{x}\right)=x\right\}$.

Let $y \in O \backslash\{x\}$ be such that $R_{s^{\prime}}: y x \emptyset \ldots$ and $\left.R_{s^{\prime}}\right|_{O \backslash\{y\}}=\left.R_{s^{\prime}}^{x}\right|_{O \backslash\{y\}}$, i.e., we obtain $R_{s^{\prime}}$ from $R_{s^{\prime}}^{x}$ by moving object $y$ on top of agent $s^{\prime}$ 's preferences. By unavailable-type-invariance,

$$
\varphi\left(R_{S \backslash\left\{s^{\prime}\right\}}^{x}, R_{N \backslash S}^{\emptyset}, R_{s^{\prime}}, l_{x}\right)=\varphi\left(R_{S}^{x}, R_{N \backslash S}^{\emptyset}, l_{x}\right) .
$$

We now add object $y$. Let $l_{x} 1_{y}$ denote the capacity vector where $l$ copies of object $x$ are available and one copy of object $y$ (and no copies of any other object). By resource-monotonicity and $\varphi_{s}\left(R_{S \backslash\left\{s^{\prime}\right\}}^{x}, R_{N \backslash S}^{\emptyset}, R_{s^{\prime}}, l_{x}\right)=\varphi_{s}\left(R_{S}^{x}, R_{N \backslash S}^{\emptyset}, l_{x}\right)=x$, we have $\varphi_{s}\left(R_{S \backslash\left\{s^{\prime}\right\}}^{x}, R_{N \backslash S}^{\emptyset}, R_{s^{\prime}}, l_{x} 1_{y}\right)=x$. By weak non-wastefulness and individual-rationality, $\varphi_{s^{\prime}}\left(R_{S \backslash\left\{s^{\prime}\right\}}^{x}, R_{N \backslash S}^{\emptyset}, R_{s^{\prime}}, 1_{y}\right)=y$ which, together with resource-monotonicity, implies $\varphi_{s^{\prime}}\left(R_{S \backslash\left\{s^{\prime}\right\}}^{x}, R_{N \backslash S}^{\emptyset}, R_{s^{\prime}}, l_{x} 1_{y}\right)=y$.

Next, let $\hat{R}_{s^{\prime}} \in \mathcal{R}$ be such that $\hat{R}_{s^{\prime}}: y \emptyset x \ldots$, and $\left.\hat{R}_{s^{\prime}}\right|_{O}=\left.R_{s^{\prime}}\right|_{O}$, i.e., we obtain $\hat{R}_{s^{\prime}}$ from $R_{s^{\prime}}$ by moving the null object $\emptyset$ between objects $y$ and $x$. Note that $\hat{R}_{s^{\prime}}$ is a truncation of $R_{s^{\prime}}$. Hence, by truncation-invariance,

$$
\varphi_{s}\left(R_{S \backslash\left\{s^{\prime}\right\}}^{x}, R_{N \backslash S}^{\emptyset}, \hat{R}_{s^{\prime}}, l_{x} 1_{y}\right)=x \text { and } \varphi_{s^{\prime}}\left(R_{S \backslash\left\{s^{\prime}\right\}}^{x}, R_{N \backslash S}^{\emptyset}, \hat{R}_{s^{\prime}}, l_{x} 1_{y}\right)=y .
$$

We now remove (the added) object $y$. By individual-rationality, $\varphi_{s^{\prime}}\left(R_{S \backslash\left\{s^{\prime}\right\}}^{x}, R_{N \backslash S}^{\emptyset}, \hat{R}_{s^{\prime}}, l_{x}\right)=\emptyset$ and $\varphi_{s}\left(R_{S \backslash\left\{s^{\prime}\right\}}^{x}, R_{N \backslash S}^{\emptyset}, \hat{R}_{s^{\prime}}, l_{x}\right) \in\{x, \emptyset\}$. If $\varphi_{s}\left(R_{S \backslash\left\{s^{\prime}\right\}}^{x}, R_{N \backslash S}^{\emptyset}, \hat{R}_{s^{\prime}}, l_{x}\right)=\emptyset$, then by weak nonwastefulness and individual-rationality there exists $s^{\prime \prime} \in S$ with preferences $R_{s^{\prime \prime}}^{x}$ such that $\varphi_{s^{\prime \prime}}\left(R_{S \backslash\left\{s^{\prime}\right\}}^{x}, R_{N \backslash S}^{\emptyset}, \hat{R}_{s^{\prime}}, l_{x}\right)=x$ and $\varphi_{s^{\prime \prime}}\left(R_{S \backslash\left\{s^{\prime}\right\}}^{x}, R_{N \backslash S}^{\emptyset}, \hat{R}_{s^{\prime}}, l_{x} 1_{y}\right)=\emptyset$; contradicting resourcemonotonicity. Thus, $\varphi_{s}\left(R_{S \backslash\left\{s^{\prime}\right\}}^{x}, R_{N \backslash S}^{\emptyset}, \hat{R}_{s^{\prime}}, l_{x}\right)=x$. By unavailable-type-invariance,

$$
\varphi\left(R_{S \backslash\left\{s^{\prime}\right\}}^{x}, R_{N \backslash S}^{\emptyset}, \hat{R}_{s^{\prime}}, l_{x}\right)=\varphi\left(R_{S \backslash\left\{s^{\prime}\right\}}^{x}, R_{(N \backslash S) \cup\left\{s^{\prime}\right\}}^{\emptyset}, l_{x}\right) .
$$


Hence, $\varphi_{s}\left(R_{S \backslash\left\{s^{\prime}\right\}}^{x}, R_{(N \backslash S) \cup\left\{s^{\prime}\right\}}^{\emptyset}, l_{x}\right)=x$. Thus, $s \in \mathcal{C}_{x}\left(S \backslash\left\{s^{\prime}\right\}, l\right)$.

So far we have proven that for all $x \in O, \mathcal{C}_{x}$ is acceptant and substitutable. By Lemma 1, for all $x \in O, \mathcal{C}_{x}$ is consistent. Furthermore, by resource-monotonicity, $\mathcal{C}_{x}$ is monotonic, i.e., $\mathcal{C}_{x}(S, l) \subseteq \mathcal{C}_{x}(S, l+1)$.

Let mechanism $\varphi$ satisfy strategy-proofness. With the following lemma, Theorem 2 follows.

Lemma 3. For all $(R, q) \in \mathcal{R}^{N} \times \mathcal{Q}, \varphi(R, q)$ is $\mathcal{C}$-stable.

Proof. Let $(R, q) \in \mathcal{R}^{N} \times \mathcal{Q}$. Assume that $\varphi(R, q)$ is not $\mathcal{C}$-stable.

By individual-rationality, for all $i \in N, \varphi_{i}(R, q) R_{i} \emptyset$. Since for all $x \in O_{+}(q) \cup\{\emptyset\}, \mathcal{C}_{x}$ is acceptant, we have $\mathcal{C}_{x}\left(\varphi(R, q)(x), q_{x}\right)=\varphi(R, q)(x)$ (recall that $\varphi(R, q)(x)$ denotes the set of agents that receive $x$ at $\varphi(R, q))$. Hence, (s1) in the definition of $\mathcal{C}$-stability is satisfied.

Thus, (s2) in the definition of $\mathcal{C}$-stability is violated. Then, there exists an agent-type pair $(i, x) \in N \times O_{+}(q)$ such that $x P_{i} \varphi_{i}(R, q)$ and $i \in \mathcal{C}_{x}\left(\varphi(R, q)(x) \cup\{i\}, q_{x}\right)$.

Let $\bar{R} \in \mathcal{R}^{N}$ be such that (i) for all $j \in N$ such that $\varphi_{j}(R, q) \neq \emptyset, \bar{R}_{j}$ is a truncation of $R_{j}$ such that there exists no $y \in O \backslash\left\{\varphi_{j}(R, q)\right\}$ with $\varphi_{j}(R, q) \bar{R}_{j} y \bar{R}_{j} \emptyset$ and (ii) for all $j \in N$ such that $\varphi_{j}(R, q)=\emptyset, \bar{R}_{j}=R_{j}$. (By individual-rationality, $\bar{R}_{j}$ in (i) is well-defined as truncation of $R_{j}$.) By truncation-invariance, $\varphi(\bar{R}, q)=\varphi(R, q)$ and $(i, x) \in N \times O_{+}(q)$ is such that $x \bar{P}_{i} \varphi_{i}(\bar{R}, q)$ and $i \in \mathcal{C}_{x}\left(\varphi(\bar{R}, q)(x) \cup\{i\}, q_{x}\right)$.

Let $T=\left\{j \in N: x \bar{P}_{j} \varphi_{j}(\bar{R}, q)\right\}$. Note that $i \in T$. Let $l_{q_{x}}$ denote the capacity vector where $q_{x}$ copies of $x$ are available and no copy of any other real object is available.

If for $j \in T, \varphi_{j}\left(\bar{R}, l_{q_{x}}\right)=x$, then by resource-monotonicity, $\varphi_{j}(\bar{R}, q) \bar{R}_{j} x$, contradicting $x \bar{P}_{j}$ $\varphi_{j}(\bar{R}, q)$. Hence, for all $j \in T, \varphi_{j}\left(\bar{R}, l_{q_{x}}\right)=\emptyset$. If $\left|\left\{j \in N \backslash T: x \bar{P}_{j} \emptyset\right\}\right|<q_{x}$, then this contradicts weak non-wastefulness. Thus, $T \subsetneq S:=\left\{j \in N: x \bar{P}_{j} \emptyset\right\}$ and $|S| \geq|T|+q_{x}$.

By construction of $\bar{R}$, for all $j \in N$ we have either (i) $x \bar{P}_{j} \varphi_{j}(\bar{R}, q)$ (and $j \in T$ ) or (ii) $\varphi_{j}(\bar{R}, q)=x$ or (iii) $\emptyset \bar{P}_{j} x$. Then, (i), (ii), and individual-rationality imply $|S|=|T|+q_{x}$ and there exist distinct $k_{1}, \ldots, k_{q_{x}} \in N \backslash T$ such that $S=T \cup\left\{k_{1}, \ldots, k_{q_{x}}\right\}$ and $\varphi_{k_{1}}(\bar{R}, q)=\cdots=\varphi_{k_{q_{x}}}(\bar{R}, q)=x$. In other words, $\varphi(\bar{R}, q)(x)=\left\{k_{1}, \ldots, k_{q_{x}}\right\}$.

Next, the fact that for all $j \in T, \varphi_{j}\left(\bar{R}, l_{q_{x}}\right)=\emptyset$, together with weak non-wastefulness and individual-rationality, implies that $\varphi_{k_{1}}\left(\bar{R}, l_{q_{x}}\right)=\cdots=\varphi_{k_{q_{x}}}\left(\bar{R}, l_{q_{x}}\right)=x$. By unavailabletype-invariance, $\varphi\left(\left(R_{S}^{x}, R_{N \backslash S}^{\emptyset}\right), l_{q_{x}}\right)=\varphi\left(\bar{R}, l_{q_{x}}\right)$. Hence, $\mathcal{C}_{x}\left(S, q_{x}\right)=\left\{k_{1}, \ldots, k_{q_{x}}\right\}$. By substitutability, $\mathcal{C}_{x}\left(\left\{k_{1}, \ldots, k_{q_{x}}, i\right\}, q_{x}\right)=\left\{k_{1}, \ldots, k_{q_{x}}\right\}$; contradicting $i \in \mathcal{C}_{x}\left(\varphi(\bar{R}, q)(x) \cup\{i\}, q_{x}\right)=$ $\mathcal{C}_{x}\left(\left\{k_{1}, \ldots, k_{q_{x}}, i\right\}, q_{x}\right)$.

So far we have established that for any mechanism $\varphi$ that satisfies the properties of Theorem 2 , there exists a choice structure $\mathcal{C}$ (where the choice structure satisfies acceptance, monotonicity, substitutability, and consistency) such that for any $(R, q) \in \mathcal{R}^{N} \times \mathcal{Q}, \varphi(R, q)$ is $\mathcal{C}$-stable. Hence, in 
the terminology of two-sided matching, the mechanism $\varphi$ picks a stable matching for the many-toone market where types have preferences over agents who consume the types based on the choice structure $\mathcal{C}$ and agents have strict preferences over types based on preferences $R$ (see Hatfield and Milgrom, 2005). For these markets it is well-known that the choice-deferred-acceptance-mechanism is the only strategy-proof stable matching mechanism. Hence, Theorem 3 follows immediately from Theorem 2.

\section{A.5 Independence of Properties in Theorem 3 and Corollary 6}

We denote a problem by $(R, q)$ where $R \in \mathcal{R}^{S}(S \in \mathcal{N})$ and $q \in \mathcal{Q}$. We consider variable-resource and variable-population mechanisms $\varphi:\left(\cup_{S \in \mathcal{N}} \mathcal{R}^{S}\right) \times \mathcal{Q} \rightarrow \mathcal{A}$. Without loss of generality we assume $N=\{1, \ldots, n\}$ to establish independence.

For any strict order $\pi$ of agents in $N$, we denote the corresponding serial dictatorship mechanism by $f^{\pi}$; for example, if $\pi: 12 \ldots(n-1) n$, then $f^{\pi}$ works as follows: for each problem $R \in \mathcal{R}^{S}(S \in \mathcal{N})$, first agent $\min S$ chooses his preferred object in $O_{+}(q) \cup\{\emptyset\}$, then agent $\min (S \backslash\{\min S\})$ chooses his preferred object among the remaining available objects (respecting the capacity constraints of $q$ ), etc. Note that for each strict order $\pi$ of $N, f^{\pi}=D A^{\succ^{\pi}}$ where $\succ^{\pi}$ equals the strict order $\pi$, i.e. for all $x \in O, \succ_{x}^{\pi}=\pi$. Thus, each serial dictatorship mechanism $f^{\pi}$ satisfies unavailable-type-invariance, weak non-wastefulness, resource-monotonicity, population-monotonicity, truncation-invariance, and strategy-proofness.

The following examples establish the independence of the properties (properties not mentioned in the examples follow easily) in Corollary 6 and Theorem 3 (by restricting any mechanism to the domain $\left.\mathcal{R}^{N} \times \mathcal{Q}\right)$.

Not unavailable-type-invariant: Let $N=\{1,2,3\}, O=\{x, y\}$, and there are at most two copies of $x$ and at most one copy of $y$ available, i.e., $\bar{q}_{x}=2$ and $\bar{q}_{y}=1$.

Let $\succ_{x}: 123, \succ_{x}^{\prime}: 132$, and $\succ_{y}: 123$. Let $\succ=\left(\succ_{x}, \succ_{y}\right)$ and $\succ^{\prime}=\left(\succ_{x}^{\prime}, \succ_{y}\right)$. Then, for each $S \in \mathcal{N}$ and each problem $(R, q) \in \mathcal{R}^{S} \times \mathcal{Q}$,

$$
\varphi(R, q)= \begin{cases}D A^{\succ}(R, q) & \text { if } q_{y}=0,1 \in S, \text { and } R_{1}: y x \emptyset \text { and } \\ D A^{\succ^{\prime}}(R, q) & \text { otherwise. }\end{cases}
$$

Note that if for $R \in \mathcal{R}^{N}$ we have $R_{1}: y x \emptyset, A\left(R_{2}\right)=A\left(R_{3}\right)=\{x\}$, and $q_{x}=2$ and $q_{y}=0$, then $\varphi_{1}(R, q)=x=\varphi_{2}(R, q)$ and $\varphi_{3}(R, q)=\emptyset$ whereas for $R_{1}^{\prime} \in \mathcal{R}$ with $A\left(R_{1}^{\prime}\right)=\{x\}$ and $R^{\prime}=$ $\left(R_{1}^{\prime}, R_{-1}\right)$ we have $\varphi_{1}\left(R^{\prime}, q\right)=x=\varphi_{3}\left(R^{\prime}, q\right)$ and $\varphi_{2}\left(R^{\prime}, q\right)=\emptyset$. Thus, $\left.R\right|_{O_{+}(q) \cup\{\emptyset\}}=\left.R^{\prime}\right|_{O_{+}(q) \cup\{\emptyset\}}$ and $\varphi(R, q) \neq \varphi\left(R^{\prime}, q\right)$, i.e., $\varphi$ violates unavailable-type-invariance. Note that this argument also establishes that there exists no acceptant choice structure $\mathcal{C}$ such that $\varphi$ is $\mathcal{C}$-stable: (i) $\varphi_{1}(R, q)=$ $x=\varphi_{2}(R, q)$ and $\varphi_{3}(R, q)=\emptyset$ imply $3 \notin \mathcal{C}_{x}(\{1,2,3\}, 2)$ and (ii) $\varphi_{1}\left(R^{\prime}, q\right)=x=\varphi_{3}\left(R^{\prime}, q\right)$ and 
$\varphi_{2}\left(R^{\prime}, q\right)=\emptyset$ imply $2 \notin \mathcal{C}_{x}(\{1,2,3\}, 2)$; now (i) and (ii) yield $\left|\mathcal{C}_{x}(\{1,2,3\}, 2)\right|<2$ and $\mathcal{C}_{x}$ is not acceptant.

In order to see that $\varphi$ satisfies resource-monotonicity, let $R \in \mathcal{R}^{N}$ and $q, q^{\prime} \in \mathcal{Q}$ be such that $q \leq q^{\prime}$ (resource-monotonicity is trivially satisfied when not all agents are present). If $\varphi$ uses the same priority structure for $(R, q)$ and $\left(R, q^{\prime}\right)$, then resource-monotonicity is satisfied. Thus, $q_{y}=0<1=q_{y}^{\prime}$ and $R_{1}: y x \emptyset$. Note that the difference in $\succ_{x}$ and $\succ_{x}^{\prime}$ only matters for $R \in \mathcal{R}^{N}$ with $x \in A\left(R_{2}\right) \cap A\left(R_{3}\right)$. Then, for all the following specifications of $q$ and $q^{\prime}$ we see that resourcemonotonicity is satisfied (see Table 4 below).

\begin{tabular}{|c|c|c|c|}
\hline$q=\left(q_{y}, q_{x}\right)$ & $q^{\prime}=\left(q_{y}^{\prime}, q_{x}^{\prime}\right)$ & $\varphi(R, q)$ & $\varphi\left(R, q^{\prime}\right)$ \\
\hline \hline$(0,0)$ & $(1,0)$ & $(\emptyset, \emptyset, \emptyset)$ & $(y, \emptyset, \emptyset)$ \\
$(0,0)$ & $(1,1)$ & $(\emptyset, \emptyset, \emptyset)$ & $(y, \emptyset, x)$ \\
$(0,0)$ & $(1,2)$ & $(\emptyset, \emptyset, \emptyset)$ & $(y, x, x)$ \\
$(0,1)$ & $(1,1)$ & $(x, \emptyset, \emptyset)$ & $(y, \emptyset, x)$ \\
$(0,1)$ & $(1,2)$ & $(x, \emptyset, \emptyset)$ & $(y, x, x)$ \\
$(0,2)$ & $(1,2)$ & $(x, x, \emptyset)$ & $(y, x, x)$ \\
\hline
\end{tabular}

Table 4: Resource-Monotonicity is satisfied.

In order to see that $\varphi$ satisfies population-monotonicity, let $(R, q) \in \mathcal{R}^{N} \times \mathcal{Q}$ and $S \subseteq N$ with $|S|=2$. If $\varphi$ uses the same priority structure for $(R, q)$ and $\left(R_{S}, q\right)$, then population-monotonicity is satisfied. Thus, $q_{y}=0, R_{1}: y x \emptyset$, and $1 \notin S$. Hence, $S=\{2,3\}$. For $q_{x}=2$, populationmonotonicity is satisfied. For $q_{x}=1, \varphi_{1}(R, q)=x$ and agent 1 leaving also implies populationmonotonicity.

Not weakly non-wasteful: Let $\pi: 12 \ldots(n-1) n$. Fix a type $y \in O$ and for each $q \in \mathcal{Q}$ define $\hat{q} \in \mathcal{Q}$ such that $\hat{q}_{y}=0$ and for all $x \in O \backslash\{y\}, \hat{q}_{x}=q_{x}$. Then, for each $R \in \mathcal{R}^{S}(S \in \mathcal{N})$ and $q \in \mathcal{Q}$,

$$
\varphi(R, q)=f^{\pi}(R, \hat{q}) .
$$

Not resource-monotonic: Let $\pi$ and $\pi^{\prime}$ be two distinct strict orders of agents in $N$. Fix a capacity vector $\tilde{q} \in \mathcal{Q}$. Then, for each $R \in \mathcal{R}^{S}(S \in \mathcal{N})$ and $q \in \mathcal{Q}$,

$$
\varphi(R, q)= \begin{cases}f^{\pi}(R, q) & \text { if } q=\tilde{q} \text { and } \\ f^{\pi^{\prime}}(R, q) & \text { otherwise. }\end{cases}
$$

Not population-monotonic: Let $\pi$ and $\pi^{\prime}$ be two distinct strict orders of agents in $N$. Then, for each $R \in \mathcal{R}^{S}(S \in \mathcal{N})$ and $q \in \mathcal{Q}$,

$$
\varphi(R)= \begin{cases}f^{\pi}(R, q) & \text { if } S=N \text { and } \\ f^{\pi^{\prime}}(R, q) & \text { otherwise. }\end{cases}
$$


Not strategy-proof: Let $\succ$ be a priority structure. Then, the responsive-deferred-acceptancemechanism based on the type-optimal stable allocation that is obtained by using Gale and Shapley's (1962) type-proposing deferred-acceptance-algorithm satisfies all properties except strategyproofness.

Not truncation-invariant: Example 2 establishes the independence of truncation-invariance.

\section{A.6 Proof of Corollary 2}

*-Acyclic choice-deferred-acceptance mechanisms satisfy all the properties listed in the corollary.

(a) Let $\varphi$ be a mechanism satisfying unavailable-type-invariance, efficiency, resource-monotonicity, and truncation-invariance. Since efficiency implies weak non-wastefulness, by Theorem 2 , there exists a choice structure $\mathcal{C}$ such that $\varphi$ is $\mathcal{C}$-stable. Since the choice-deferred-acceptance-mechanism Pareto dominates any other stable mechanism (Kumano, 2009, Proposition 2), efficiency implies that $\varphi=D A^{\mathcal{C}}$. Finally, by efficiency and Kumano (2009, Theorem 1$), \mathcal{C}$ must be ${ }^{*}$-acyclic.

(b) Let $\varphi$ be a mechanism satisfying individual-rationality, weak non-wastefulness, resourcemonotonicity, and group-strategy-proofness. By Ehlers and Klaus (2004, Lemma 1), groupstrategy-proofness implies unavailable-type-invariance. Furthermore, group-strategy-proofness implies truncation-invariance and strategy-proofness. Hence, by Theorem 3, there exists a choice structure $\mathcal{C}$ such that $\varphi=D A^{\mathcal{C}}$. Finally, by group-strategy-proofness and Kumano (2009, Theorem 1$), \mathcal{C}$ must be ${ }^{*}$-acyclic.

\section{A.7 Independence of Properties in Corollary 4}

Recall that $S \in \mathcal{N}$ is fixed. Without loss of generality we assume $S=N=\{1, \ldots, n\}$ to establish independence and we consider fixed-population mechanisms $\varphi: \mathcal{R}^{N} \times \mathcal{Q}_{1} \rightarrow \mathcal{A}$. To simplify notation, we will at times denote a problem by $(R, H)$ where $H \subseteq O$ denotes the real objects that are available with capacity 1 .

For any strict order $\pi$ of agents in $N$, we denote the corresponding serial dictatorship mechanism by $f^{\pi}$; for example, if $\pi: 12 \ldots(n-1) n$, then $f^{\pi}$ works as follows: for each problem $(R, H)$, first agent 1 chooses his preferred object in $H \cup\{\emptyset\}$, then agent 2 chooses his preferred object from the remaining objects $\left(H \backslash\left\{f_{1}^{\pi}(R, H)\right\}\right) \cup\{\emptyset\}$, etc. Note that for each strict order $\pi$ of $N, f^{\pi}=D A^{\succ^{\pi}}$ where $\succ^{\pi}$ equals the strict order $\pi$, i.e., for all $x \in O, \succ_{x}^{\pi}=\pi$. Thus, each serial dictatorship mechanism $f^{\pi}$ satisfies unavailable-type-invariance, weak non-wastefulness, resource-monotonicity, truncation-invariance, and strategy-proofness.

The following examples establish the independence of the properties (properties not mentioned in the examples follow easily) in Corollary 4. 
Not unavailable-type-invariant: Let $n \geq 3$ and $\pi: 123 \ldots(n-1) n$ and $\pi^{\prime}: 1 n(n-1) \ldots 32$. Then, for each $R \in \mathcal{R}^{N}$ and $H \subseteq O(H \neq \emptyset)$,

$$
\varphi(R, H)= \begin{cases}f^{\pi}(R, H) & \text { if } A\left(R_{1}\right)=\emptyset \text { and } \\ f^{\pi^{\prime}}(R, H) & \text { otherwise. }\end{cases}
$$

Not weakly non-wasteful: Fix a type $y \in O$ and $\pi: 12 \ldots(n-1) n$. Then, for each $R \in \mathcal{R}^{N}$ and $H \subseteq O(H \neq \emptyset)$,

$$
\varphi(R, H)=f^{\pi}(R, H \backslash\{y\}) .
$$

Not resource-monotonic: ${ }^{12}$ Let $\pi$ and $\pi^{\prime}$ be two distinct strict orders of agents in $N$. Then, for each $R \in \mathcal{R}^{N}$ and $H \subseteq O(H \neq \emptyset)$,

$$
\varphi(R, H)= \begin{cases}f^{\pi}(R, H) & \text { if } H=O \text { and } \\ f^{\pi^{\prime}}(R, H) & \text { otherwise }\end{cases}
$$

Not strategy-proof: Let $\succ$ be a priority structure. Then, the responsive-deferred-acceptancemechanism based on the type-optimal stable allocation that is obtained by using Gale and Shapley's (1962) type-proposing deferred-acceptance-algorithm satisfies all properties except strategyproofness. $^{13}$

Not truncation-invariant: Let $N=\{1,2,3\}, O=\{x, y\}, \succ_{x}: 123, \succ_{x}^{\prime}: 132$, and $\succ_{y}: 123$. Let $\succ=\left(\succ_{x}, \succ_{y}\right)$ and $\succ^{\prime}=\left(\succ_{x}^{\prime}, \succ_{y}\right)$. Then, for each $R \in \mathcal{R}^{N}$ and $H \subseteq O(H \neq \emptyset)$,

$$
\varphi(R, H)= \begin{cases}D A^{\succ}(R, H) & \text { if } \emptyset P_{1} x \text { and } x \in H \text { and } \\ D A^{\succ^{\prime}}(R, H) & \text { otherwise. }\end{cases}
$$

Let $R_{1}: y \emptyset x, R_{2}: x \emptyset y, R_{3}: x \emptyset y$, and $R_{1}^{\prime}: y x \emptyset$. Let $R=\left(R_{1}, R_{2}, R_{3}\right)$ and $R^{\prime}=\left(R_{1}^{\prime}, R_{2}, R_{3}\right)$. Note that $R_{1}$ is a truncation of $R_{1}^{\prime}$ and $\varphi_{1}(R,\{x, y\})=y=\varphi_{1}\left(R^{\prime},\{x, y\}\right)$. However, $\varphi_{2}(R,\{x, y\})=$ $x$ and $\varphi_{3}\left(R^{\prime},\{x, y\}\right)=x$, i.e., $\varphi$ violates truncation-invariance. Next, we show strategy-proofness and resource-monotonicity for this mechanism.

For strategy-proofness, note that agents 2 and 3 cannot change the priority structure by reporting a false preference relation. Agent 1 always receives his most preferred object in $H$ for any problem $(R, H)$. Thus, agent 1 cannot profitably manipulate by reporting a false preference relation.

For resource-monotonicity, let $|H|=1$ and $R \in \mathcal{R}^{N}$. If there is a violation of resourcemonotonicity, then $\varphi$ must use different priority structures for $(R, H)$ and $(R,\{x, y\})$. But then we must have $H=\{y\}$ and both $\varphi(R,\{y\})=D A^{\succ^{\prime}}(R,\{y\})$ and $\varphi(R,\{x, y\})=D A^{\succ}(R,\{x, y\})$.

\footnotetext{
${ }^{12}$ Note that here we could have chosen the TTC-mechanism as an example satisfying all properties in Theorem 4 except for resource-monotonicity.

${ }^{13}$ Given $\succ$, there exist many "artificial" stable mechanisms satisfying all properties except strategy-proofness. For instance, let $v \in\{1, \ldots,|O|-1\}$. For all $R \in \mathcal{R}^{N}$ and $H \subseteq O(H \neq \emptyset)$, let the mechanism $\phi$ choose the type-optimal stable allocation (for $\succ$ ) if $|H| \leq v$ and the agent-optimal stable allocation (for $\succ$ ) if $|H|>v$. The mechanism $\phi$ satisfies all properties except strategy-proofness.
} 
If $y P_{1} \emptyset$, then $\varphi_{1}(R,\{y\})=y$ and $\varphi_{2}(R,\{y\})=\varphi_{3}(R,\{y\})=\emptyset$, and all agents weakly prefer $\varphi(R,\{x, y\})$ to $\varphi(R,\{y\})$.

If $\emptyset P_{1} y$, then $\varphi_{1}(R, y)=\emptyset$. Since $\varphi(R,\{x, y\})=D A^{\succ}(R,\{x, y\})$, we have $\emptyset P_{1} x$ and by individual-rationality, $\varphi_{1}(R,\{x, y\})=\emptyset$. Note that $2 \succ_{x} 3,2 \succ_{y} 3$, and $2 \succ_{y}^{\prime} 3$ (the latter because $\left.\succ_{y}=\succ_{y}^{\prime}\right)$. Let $\pi: 231$. Then, $\varphi(R,\{y\})=D A^{\succ^{\prime}}(R,\{y\})=f^{\pi}(R,\{y\})$ and $\varphi(R,\{x, y\})=$ $D A^{\succ}(R,\{x, y\})=f^{\pi}(R,\{x, y\})$. Hence, resource-monotonicity is satisfied. 